Mathematical Models and Methods in Applied Sciences

(C) World Scientific Publishing Company

\title{
Heteroclinic connections for nonlocal equations
}

\author{
Serena Dipierro \\ Department of Mathematics and Statistics, University of Western Australia, \\ 35 Stirling Highway, Crawley, WA6009, Australia \\ serena.dipierro@uwa.edu.au \\ Stefania Patrizi \\ Department of Mathematics, University of Texas at Austin, \\ 2515 Speedway Stop C1200, Austin, Texas 78712-1202, USA \\ spatrizi@math.utexas.edu \\ Enrico Valdinoci \\ Department of Mathematics and Statistics, University of Western Australia, \\ 35 Stirling Highway, Crawley, WA6009, Australia \\ enrico.valdinoci@uwa.edu.au \\ Received (Day Month Year) \\ Revised (Day Month Year) \\ Accepted (Day Month Year) \\ Communicated by (xxxxxxxxx)
}

\begin{abstract}
We construct heteroclinic orbits for a strongly nonlocal integro-differential equation. Since the energy associated to the equation is infinite in such strongly nonlocal regime, the proof, based on variational methods, relies on a renormalized energy functional, exploits a perturbation method of viscosity type, combined with an auxiliary penalization method, and develops a free boundary theory for a double obstacle problem of mixed local and nonlocal type.

The description of the stationary positions for the atom dislocation function in a perturbed crystal, as given by the Peierls-Nabarro model, is a particular case of the result presented.
\end{abstract}

Keywords: Heteroclinic solutions; Peierls-Nabarro model; crystal dislocation; fractional Laplacian; nonlocal equations

AMS Subject Classification: 70K44, 37C29, 34A08.

\section{Introduction}

Heteroclinic orbits are a classical topic in the context of dynamical systems. Not only they are trajectories that show an interesting behavior, providing a connection between two different rest positions, but they are often the "building blocks" for constructing complicated orbits, drifting from one equilibrium to another, possibly leading to a chaotic dynamics. 
The recent literature has also taken into account the case in which the "classical" differential equations are replaced by integro-differential equations. The study of these nonlocal equations is not only motivated by mathematical curiosity and by the will driving the scientists of facing with new challenging problems, but it also possesses concrete motivations in applied sciences: in particular, our main motivation for the problem treated in this paper comes from the description of the stationary positions for the atom dislocation in crystals, as provided by the PeierlsNabarro model, see e.g. 30 and Section 2 of [20]. In this context, the evolution of the dislocation function on the "slip line" (i.e., the intersection between the "slip plane", along which the crystal experiences a plastic deformation, and a transversal reference plane) is described by an equation of fractional type, as a consequence of the balance between the elastic bonds that link the atoms and the internal force of the crystals which tends to place all the atoms into a periodically organized lattice.

Concretely, in the Peierls-Nabarro model for edge dislocations, one considers equations that can be written along the slip line as

$$
\sqrt{-\Delta} Q(x)+W^{\prime}(Q(x))=0 \quad \text { for any } x \in \mathbb{R},
$$

where $W$ is a multi-well potential and the diffusion operator is the square root of the Laplacian, which (up to normalizing multiplicative constants) is the integrodifferential operator

$$
\sqrt{-\Delta} Q(x):=\mathrm{P} . \mathrm{V} . \int_{\mathbb{R}} \frac{Q(x)-Q(y)}{|x-y|^{2}} d y:=\lim _{\varrho \searrow 0} \int_{\mathbb{R} \backslash B_{\varrho}(x)} \frac{Q(x)-Q(y)}{|x-y|^{2}} d y .
$$

In the setting of 1.1 , the function $Q: \mathbb{R} \rightarrow \mathbb{R}$ represents a dislocation function (i.e., roughly speaking, a measure of the atomic disregistry with respect to the ideal rest configuration of a perfect crystal); the diffusion operator in (1.1) and (1.2) takes into account the effect on the slip line of the elastic bonds between different atoms in the crystal and the potential $W$ is induced by the large-scale pattern of the crystal itself (see e.g. 30] and Section 2 of 20] for additional details).

We also recall that, at a microscopic scale, there is a strong connection between the Peierls-Nabarro model and the Frenkel-Kontorova model, see 23]. Related models appear also in the study of the Benjamin-Ono equation, see [44], in boundary reaction equations, see [8], and in spin systems on lattices, see [1]. In addition, the study of nonlocal equations with a singular kernel is a very intense subject of research in terms of harmonic analysis, see e.g. [43], and of regularity theory, see e.g. 41.

The mathematical framework in which we work here is the following. Given a function $Q: \mathbb{R} \rightarrow \mathbb{R}$, the nonlocal operator that we take into account in this paper is given by

$$
\begin{aligned}
\mathscr{L} Q(x) & :=\mathrm{P} . \mathrm{V} \cdot \int_{\mathbb{R}}(Q(x)-Q(y)) K(x-y) d y \\
& :=\lim _{\varrho \searrow 0} \int_{\mathbb{R} \backslash B_{\varrho}(x)}(Q(x)-Q(y)) K(x-y) d y .
\end{aligned}
$$


The kernel $K$ is supposed to be even and such that

$$
\frac{\theta_{0}}{|r|^{1+2 s}} \chi_{\left[0, r_{0}\right]}(r) \leqslant K(r) \leqslant \frac{\Theta_{0}}{|r|^{1+2 s}},
$$

for some $\Theta_{0} \geqslant \theta_{0}>0$ and some $r_{0}>0$, with

$$
s \in\left(\frac{1}{4}, \frac{1}{2}\right] .
$$

Of course, the case under consideration comprises in particular the original PeierlsNabarro model in 1.2 , which corresponds to the choice

$$
s:=\frac{1}{2} \quad \text { and } \quad K(r):=\frac{1}{|r|^{2}} .
$$

In the equations that we take into account, the diffusive operator $\mathscr{L}$ is balanced by a forcing term of potential type. More precisely, we consider a non-negative multi-well potential $W \in C^{2}(\mathbb{R}, \mathbb{R})$ with a locally finite set of minima. Namely, we suppose that $W \geqslant 0$ and that there exists $\mathscr{Z} \subset \mathbb{R}$ which is a discrete set (i.e., it has no accumulation points) such that

$$
W(\zeta)=0 \text { for any } \zeta \in \mathscr{Z} \text { and } W(r)>0 \text { for any } r \in \mathbb{R} \backslash \mathscr{Z} .
$$

We also suppose that $W$ grows quadratically from its minima, that is

$$
\begin{aligned}
& c_{0}|\xi|^{2} \leqslant W(\zeta+\xi) \leqslant C_{0}|\xi|^{2}, \\
& \text { for some } C_{0}>c_{0}>0, \text { for all } \zeta \in \mathscr{Z} \text { and } \xi \in B_{\delta_{0}}, \text { with } \delta_{0}>0 .
\end{aligned}
$$

We also assume that $W$ is monotone near the equilibria, that is

$$
W^{\prime}(x)>0 \text { for all } x \in\left(\zeta, \zeta+\delta_{0}\right) \text {, and } W^{\prime}(x)<0 \text { for all } x \in\left(\zeta-\delta_{0}, \zeta\right) \text {. }
$$

In our framework, the potential is modulated by an oscillatory function $a$. This function is supposed to maintain the sign of the potential, namely we assume that

$$
a(x) \in[\underline{a}, \bar{a}] \quad \text { for all } x \in \mathbb{R},
$$

for some $\bar{a}>\underline{a}>0$.

We also assume that $a$ is "non-degenerate". More precisely, we suppose that there exist $m_{1}, m_{2} \in \mathbb{R}$ and $\omega, \theta>0$ such that

$$
m_{2}-m_{1} \geqslant 2 \omega+\theta
$$

and, for $i \in\{1,2\}$,

$$
a(x)-a(x-\theta) \geqslant \gamma \text { and } a(x)-a(x+\theta) \geqslant \gamma, \quad \text { for all } x \in\left[m_{i}-\omega, m_{i}+\omega\right] \text {, }
$$

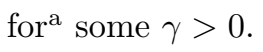

${ }^{\text {a }}$ For concreteness, we mention that the function

$$
a(x):=2+\varepsilon \cos (\delta x)
$$


In this setting, the equation that we study here has the form

$$
\mathscr{L} Q^{\star}(x)+a(x) W^{\prime}\left(Q^{\star}(x)\right)=0 \quad \text { for all } x \in \mathbb{R} .
$$

Of course, when $\mathscr{L}$ is replaced by the classical second order differential operator, equation (1.13) may be seen as a pendulum-like equation.

The main objective of this paper is to construct heteroclinic solutions of $(1.13)$, i.e. orbits which connect two different equilibria. The equilibria that we connect are the "closest" possible, as specified by the following definition:

Definition 1.1. We say that $\zeta_{1} \neq \zeta_{2} \in \mathscr{Z}$ are nearest neighbors if the open segment joining $\zeta_{1}$ and $\zeta_{2}$ does not contain any point of $\mathscr{Z}$.

Then, in this setting, our main result on the existence of heteroclinics for equation 1.13 is the following:

Theorem 1.1. Let $\zeta_{1} \in \mathscr{Z}$ and let $\zeta_{2} \in \mathscr{Z} \backslash\left\{\zeta_{1}\right\}$ be a nearest neighbor of $\zeta_{1}$.

Then, there exists a solution $Q^{\star}: \mathbb{R} \rightarrow\left[\min \left\{\zeta_{1}, \zeta_{2}\right\}, \max \left\{\zeta_{1}, \zeta_{2}\right\}\right]$ of (1.13) such that

$$
\lim _{x \rightarrow-\infty} Q^{\star}(x)=\zeta_{1} \quad \text { and } \quad \lim _{x \rightarrow+\infty} Q^{\star}(x)=\zeta_{2} .
$$

From the physical point of view, Theorem 1.1 states that an arbitrarily small perturbation of the global crystal configuration (as induced by the perturbation function $a$ ) can lead to a nontrivial displacements of the atoms of the crystal.

We observe that Theorem 1.1 is new even in the model case of the square root of the Laplacian (as described by (1.2) and (1.6)). In particular, Theorem 1.1 is new even for the original perturbed Peierls-Nabarro model. The proof that we perform is more general and it comprises several other cases of interest, and it is rather complicated, but it does not enjoy significant simplifications in the particular case of Peierls-Nabarro, and this is the main reason for which we deal with equation 1.13 here in its full generality.

with $\varepsilon, \delta \in(0,1]$ satisfies 1.12 with $m_{1}:=0, m_{2}:=\frac{2 \pi}{\delta}, \omega:=\frac{\pi}{4 \delta}, \theta:=\frac{\pi}{\delta}$ and $\gamma:=\sqrt{2} \varepsilon$. Indeed, in this case,

$$
\begin{aligned}
& \inf _{x \in\left[m_{1}-\omega, m_{1}+\omega\right] \cup\left[m_{2}-\omega, m_{2}+\omega\right]} a(x)-a(x \pm \theta) \\
= & \inf _{x \in\left[-\frac{\pi}{4 \delta}, \frac{\pi}{4 \delta}\right] \cup\left[\frac{2 \pi}{\delta}-\frac{\pi}{4 \delta}, \frac{2 \pi}{\delta}+\frac{\pi}{4 \delta}\right]} \varepsilon(\cos (\delta x)-\cos (\delta x \pm \delta \theta)) \\
= & \inf _{y \in\left[-\frac{\pi}{4}, \frac{\pi}{4}\right]} \varepsilon(\cos y-\cos (y \pm \pi)) \\
= & 2 \inf _{y \in\left[-\frac{\pi}{4}, \frac{\pi}{4}\right]} \varepsilon \cos y \\
= & \sqrt{2} \varepsilon .
\end{aligned}
$$

This example shows that there exist "small and slow perturbations of constant functions" that satisfy 1.12 . 
The advantage of keeping such a generality is that equation 1.13 also applies to models which go beyond atom dislocation in crystals. For instance, similar equations appear in the dynamics of biological populations. In this case a logistic nonlinearity is coupled to a diffusive regime, and several experiments have confirmed the tendency of many biological species to exploit nonlocal and fractional diffusive strategies, see 24, 45. The experiments on the different biological species have been also fruitfully compared and confirmed by numerical simulations, see [36. From the mathematical point of view, anomalous diffusion can provide concrete advantages for the structural organization of a biological species in terms of resources consumption (see 9, 26, 28]), it can be related to the optimal distribution of predators in an environment with sparse prays (see 42 ), and can exhibit different stability patterns (see 12, 14]). Related nonlocal equations also naturally appear in genetics, see e.g. 22 and the references therein.

Hence, in view of its fascinating mathematical framework, and due to the great source of concrete applications in physics, material sciences, ethology and genetics, we think it is worth to consider equation 1.13 in its generality, since the analytical difficulties do not get simplified by reducing to the classical Peierls-Nabarro model, and the additional generality allows us to comprise many cases of concrete interest in applied sciences.

We also point out that, differently from the classical case, the asymptotic expression in $(1.14)$ is not an immediate consequence of fractional energy estimates since, when $s \in\left(0, \frac{1}{2}\right]$, functions in $H^{s}(\mathbb{R})$ are not necessarily infinitesimal at infinity (see e.g. Appendix Appendix A for a simple example of this important phenomenon).

We remark that, on the one hand, the non-degeneracy condition in 1.12 introduces an additional difficulty to the problem, making it not invariant under translations. On the other hand, the existence of heteroclinic solutions in the homogeneous setting is known at least in some cases, see $7,8,13,17,31$. From the technical point of view, the function $a$ produces an important difference with respect to the previous literature on the subject, since the translation invariance of the system implies the monotonicity of the heteroclinic, which in turn implies a series of analytic estimates on the energy functional and allows one to use of more direct minimization principles (see $7,13,31$ for further details).

In general, our perspective is that not only the non-degeneracy condition in 1.12 provides a useful technical tool for the construction of heteroclinic solutions in problems that are not invariant under translations, but also it is an essential ingredient to deal with homoclinic and multibump orbits. As a matter of fact, as a future project we aim at using the heteroclinic connections constructed here as a "building block" for these more general types of trajectories (and, for instance, homoclinic solutions do not exist when $a$ is constant, see 7 in case of even solutions, and 15 for the general case, therefore condition 1.12 cannot be removed when dealing with homoclinic connections). 
From the physical point of view, thinking about dislocation models, the function $a$ can be considered as an arbitrarily small perturbation (and with oscillations having arbitrarily small frequencies, see the footnote on page 3 of the structural potential $W$ which is induced by the "large scale" pattern of the crystal. In a sense, one can think that an appropriate external force can slightly, and suitably, modify the perfect periodic structure of the material, thus producing this perturbed potential configuration.

As a matter of fact, on the one hand Theorem 1.1 is not necessarily a perturbation result, since it also comprises cases in which the function $a$ is not a perturbation of the constant; on the other hand, Theorem 1.1 includes the perturbative setting as a special case, and, in this sense, we can also consider Theorem 1.1 as a "structural stability" result which ensures the conservation of heteroclinic orbits under a (suitably nondegenerate) perturbation.

Furthermore, the construction of heteroclinic orbits for ordinary differential equations is a well-studied topic in the literature and, in this sense, Theorem 1.1 here is a nonlocal counterpart of some of the celebrated results obtained in 32 35 for ordinary differential equations and Hamiltonian systems. Of course, the case of nonlocal equations is conceptually quite different from that of ordinary differential equations, since usual "glueing" and "cut-and-paste" methods are not available, due to far-away energy interactions. We refer to [6] for a general introduction to nonlocal problems, also motivated from water wave models, phase transitions, material sciences and biology.

A result similar to Theorem 1.1 when the nonlocal parameter $s$ lies in the range $\left(\frac{1}{2}, 1\right)$ has been obtained in 21], see also [16. In case of homogeneous media (i.e., when $a$ is constant), heteroclinic connections corresponding to parameter ranges $s \in\left(0, \frac{1}{2}\right]$ have been studied in $7,13,31$ by energy renormalization methods.

Concerning the nonlocal parameter range considered in this paper, we recall that the case $s \in\left(0, \frac{1}{2}\right)$ can present several technical and conceptual differences with respect to the case $s \in\left(\frac{1}{2}, 1\right)$ (the case $s=\frac{1}{2}$ being typically "in between" the two cases). For instance, as shown in [11,38, several fractional equations corresponding to the parameter range $s \in\left[\frac{1}{2}, 1\right)$ present a "local behavior" at a large scale, while they preserve a "nonlocal behavior" at any scale when $s \in\left(0, \frac{1}{2}\right)$.

In our setting, to deal with the case $s \in\left(\frac{1}{4}, \frac{1}{2}\right]$ we will adopt a strategy that has been also very recently used in 13 and based on two basic steps:

- Given $\zeta_{1}, \zeta_{2} \in \mathscr{Z}$, we take a function

$$
Q_{\zeta_{1}, \zeta_{2}}^{\sharp} \in C^{\infty}\left(\mathbb{R},\left(\min \left\{\zeta_{1}, \zeta_{2}\right\}, \max \left\{\zeta_{1}, \zeta_{2}\right\}\right)\right)
$$

to be such that $Q_{\zeta_{1}, \zeta_{2}}^{\sharp}(x)=\zeta_{1}$ for any $x \in(-\infty,-1)$ and $Q_{\zeta_{1}, \zeta_{2}}^{\sharp}(x)=\zeta_{2}$ for any $x \in(1,+\infty)$. Then, we consider a renormalized energy functional 
of the form

$$
\begin{aligned}
& I_{0}(Q):=\int_{\mathbb{R}} a(x) W(Q(x)) d x \\
& +\frac{1}{4} \iint_{\mathbb{R} \times \mathbb{R}}\left(|Q(x)-Q(y)|^{2}-\left|Q_{\zeta_{1}, \zeta_{2}}^{\sharp}(x)-Q_{\zeta_{1}, \zeta_{2}}^{\sharp}(y)\right|^{2}\right) K(x-y) d x d y .
\end{aligned}
$$

The device of dealing with a renormalized energy is needed in order to avoid the divergence of the energy due to nonlocal effects in this parameter range. We stress that this energy divergence is unavoidable, since, for instance, one can easily check that the fractional Sobolev (or Aronszajn-GagliardoSlobodeckij) seminorm in $H^{s}(-R, R)$ of a smooth function connecting two constants goes like $\log R$ when $s=\frac{1}{2}$, and like $R^{1-2 s}$ when $s \in\left(0, \frac{1}{2}\right)$, thus diverging as $R \rightarrow+\infty$.

- We will perturb the original energy functional by a classical Dirichlet energy. This step is very convenient, since it allows to deal with continuous trajectory in a perturbed setting (notice that, when $s \in\left(0, \frac{1}{2}\right]$, functions in $H^{s}(\mathbb{R})$ are not necessarily continuous, see e.g. Appendix Appendix A for a simple example). After dealing with a minimization argument for such perturbed energy functional, we will obtain uniform estimates that will allow us to pass to the limit.

Furthermore, in our setting, we exploit an additional penalization method in $L^{2}(\mathbb{R})$, which has the technical advantage of localizing the transition sufficiently close to a prescribed position (say, the origin).

A series of analytical techniques coming from elliptic partial differential equations are also crucially exploited in our proofs:

- We will make use of viscosity solution methods in order to obtain regularity theories that are uniform in the perturbation parameter related to the Dirichlet energy (this is a fundamental step in order to "remove" the "local and elliptic energy perturbation" in the limit).

- We will study a double obstacle problem of mixed local and nonlocal type, which arises from the constrained minimization of the energy functional (this step is crucial in order to estimate "how the orbits separates from the constraints").

- The estimates obtained on the penalized equation will then be sufficiently stable to remove the additional penalization term.

In general, we believe that a very interesting feature provided by the equations related to the Peierls-Nabarro model lies in the fact that their complete understanding requires a synergic combination of resources and methods coming from different specific backgrounds, which include, among the others, mathematical physics, calculus of variations, partial differential equations, free boundary problems, geometric measure theory, harmonic analysis and the theory of pseudodifferential operators. 
The parameter range considered in this paper has also a special energy feature. Namely, while the interaction energy of fractional Sobolev type of a heteroclinic connection is divergent, the part coming from the potential is typically finite under assumption (1.5). To check this, we recall formula (12) in 31, according to which a heteroclinic orbit $Q(x)$ converges to the equilibrium in the homogeneous case like $\frac{\text { const }}{1+|x|^{2 s}}$. Since, by $(1.8)$, the potential $W$ is quadratic near the equilibria, the potential energy term of such trajectory behaves like

$$
\int_{\mathbb{R}} \frac{\text { const }}{\left(1+|x|^{2 s}\right)^{2}} d x
$$

which is finite when $s$ lies above the threshold $1 / 4$.

For this reason, when $s$ lies below $1 / 4$, it could be expected that a second energy renormalization is needed in order to apply variational methods (e.g. in the approach given by formula (13) in 31]) and we plan to explore this parameter range in future works.

The rest of the paper is organized as follows. In Section 2, we fix some notation, to be used in the rest of the paper. In Section 3 , we give two elementary proofs establishing a uniform bound for a nonlocal equation and a regularity result for a perturbed problem (in our setting, such bound is important to obtain uniform estimates in a perturbed problem, and the regularity result is useful to estimate errors in the "cut-and-paste" procedures).

The proof of Theorem 1.1 is then developed in Sections 413 More precisely, Section 4 is devoted to an energy estimate from below. In our setting, this bound is important to guarantee the necessary compactness for the direct methods of the calculus of variations.

Then, we exploit these variational methods to construct the heteroclinic connections, by proceeding step by step. First, in Section 5 we consider a constrained and perturbed problem. The additional perturbation provides the technical advantage that all the orbits with finite energy are in fact continuous, and this fact will allow us to make use of geometric arguments in the analysis of such orbits. The constrain is also useful to "force" the orbits close to the equilibria at infinity. As a matter of fact, in Section 6, using a double obstacle problem approach, we show that constrained minimizers are continuous with uniform bounds.

Interestingly, this obstacle problem is also of mixed local and nonlocal type, and this is a class of problems rarely studied in the existing literature. For our goals, the achievement of uniform estimates for this problem is crucial in order to have precise information when the orbit touches the variational constraints.

Also, in Sections 7 and 8 we recall the notions of clean intervals and clean points, and we prove some stickiness properties of the energy minimizers.

In Section 9, we reduce the proof of our main result to the case in which the set of equilibria consists of two points. Then, in Section 10, by taking the asymptotic constraints "far enough", we will produce a free, i.e. unconstrained, minimizer. 
In Section 11, by using estimates that are uniform with respect to the viscous perturbative parameter, we will be able to remove the viscosity perturbation.

Interestingly, the whole variational scheme presented so far is improved by an additional penalization term. This technical device is convenient to "localize" the transition of the minimal heteroclinic: to obtain the desired solution, one needs to remove the penalization term. To this end, one needs to obtain spatial asymptotics at infinity, in order to detect the limit behavior of the desired heteroclinics. For this, it is also useful to observe that solutions that are asymptotically confined near an equilibrium are in fact converging to it: this observation is contained in Section 12 .

With this, the penalized term in the equation will be removed in Section 13 . thus completing the proof of Theorem 1.1 .

\section{Notation}

- Given $I, J \subseteq \mathbb{R}$ and $f, g: \mathbb{R} \rightarrow \mathbb{R}$, we set

$$
\mathscr{B}_{I, J}(f, g):=\iint_{I \times J}(f(x)-f(y))(g(x)-g(y)) K(x-y) d x d y,
$$

and

$$
E_{I \times J}(f):=\iint_{I \times J}\left(|f(x)-f(y)|^{2}-\left|Q_{\zeta_{1}, \zeta_{2}}^{\sharp}(x)-Q_{\zeta_{1}, \zeta_{2}}^{\sharp}(y)\right|^{2}\right) K(x-y) d x d y .
$$

Notice that

$$
\begin{aligned}
\mathscr{B}_{J, I}(f, g)=\iint_{J \times I}(f(x)-f(y))(g(x)-g(y)) K(x-y) d x d y \\
\quad=\iint_{I \times J}(f(y)-f(x))(g(y)-g(x)) K(y-x) d y d x=\mathscr{B}_{I, J}(f, g),
\end{aligned}
$$

since $K$ is even. Similarly,

$$
E_{I \times J}(f)=E_{J \times I}(f) .
$$

We will also use the notation

$$
E_{I^{2}}(f)=E_{I \times I}(f) .
$$

- Given $X, Y \subseteq \mathbb{R}$, we set

$$
[v]_{K, X \times Y}:=\sqrt{\iint_{X \times Y}|v(x)-v(y)|^{2} K(x-y) d x d y} .
$$

- The Lebesgue measure of a set $A$ will be denoted by $|A|$. 


\section{A uniform bound and a regularity result for a nonlocal equation}

In this section, $\mathscr{L} u$ denotes the differential operator defined on smooth bounded functions as follows

$$
\begin{aligned}
\mathscr{L} u(x) & :=\mathrm{P} . \mathrm{V} \cdot \int_{\mathbb{R}^{N}}(u(x)-u(y)) K(x-y) d y \\
& :=\lim _{\varrho \searrow 0} \int_{\mathbb{R}^{N} \backslash B_{\varrho}(x)}(u(x)-u(y)) K(x-y) d y,
\end{aligned}
$$

where $K$ is an even kernel such that

$$
\frac{\theta_{0}}{|r|^{N+2 s}} \chi_{\left[0, r_{0}\right]}(r) \leqslant K(r) \leqslant \frac{\Theta_{0}}{|r|^{N+2 s}},
$$

for some $\Theta_{0} \geqslant \theta_{0}>0$ and some $r_{0}>0$, with $s \in(0,1)$. Of course, the setting in 1.3 is comprised here with $N:=1$.

The next is a uniform regularity result dealing with a perturbed problem:

Lemma 3.1. Let $\eta \in[0,1], s \in(0,1)$ and $f_{1}, f_{2} \in \mathbb{R}$. Let $u \in L^{\infty}\left(\mathbb{R}^{N}\right) \cap C\left(B_{1}\right)$ be a viscosity subsolution of

$$
-\eta \Delta u+\mathscr{L} u+f_{1}=0 \quad \text { in } B_{1}
$$

and a viscosity supersolution of

$$
-\eta \Delta u+\mathscr{L} u+f_{2}=0 \quad \text { in } B_{1} .
$$

Then, $u \in C^{0, \alpha}\left(B_{1 / 2}\right)$ for any $\alpha<\min \{2 s, 1\}$ and

$$
[u]_{C^{0, \alpha}\left(B_{1 / 2}\right)} \leqslant C\left(f_{2}-f_{1}+\|u\|_{L^{\infty}\left(\mathbb{R}^{N}\right)}\right)^{\frac{\alpha}{2 s}}\|u\|_{L^{\infty}\left(\mathbb{R}^{N}\right)}^{1-\frac{\alpha}{2 s}},
$$

for some $C>0$ independent of $\eta$.

Proof. We use appropriate techniques from the theory of regularity of viscosity solutions of uniformly elliptic second-order local operators, see 25, and recently extended to nonlocal operators, see e.g. 3, 29, adapted to our context. Let us introduce the following notation: given $r>0$, for a function $\phi$ we define

$$
\mathscr{L}^{1, r} \phi(x):=\int_{\{|z| \leqslant r\}}(\phi(x)-\phi(x+z)+\nabla \phi(x) \cdot z) K(z) d z
$$

and

$$
\mathscr{L}^{2, r} \phi(x):=\int_{\{|z| \geqslant r\}}(\phi(x)-\phi(x+z)) K(z) d z .
$$

Then,

$$
\mathscr{L} \phi(x)=\mathscr{L}^{1, r} \phi(x)+\mathscr{L}^{2, r} \phi(x) .
$$

We let $\phi \in C^{\infty}\left(\mathbb{R}^{N} ; \mathbb{R}_{0}^{+}\right) \cap W^{2, \infty}\left(\mathbb{R}^{N}\right)$ be such that $\phi(x)=0$ for all $x \in B_{1 / 2}$ and $\phi(x) \geqslant 1$ for all $x \in \mathbb{R}^{N} \backslash B_{3 / 4}$. We then define

$$
\psi(x):=2\|u\|_{L^{\infty}\left(\mathbb{R}^{N}\right)} \phi(x) .
$$


Since $\phi \equiv 0$ in $B_{1 / 2}$, to prove that $u \in C^{0, \alpha}\left(B_{1 / 2}\right)$ for any $\alpha<2 s$, it is enough to show that given any $\alpha<2 s$, with $\alpha \in(0,1)$, there exists $L>0$ such that, for all $x_{1}, x_{2} \in \mathbb{R}^{N}$,

$$
u\left(x_{1}\right)-u\left(x_{2}\right)-L\left|x_{1}-x_{2}\right|^{\alpha}-\psi\left(x_{1}\right) \leqslant 0 .
$$

We argue by contradiction, assuming that (3.7) does not hold true. For $\varepsilon>0$, let $u^{\varepsilon}$ and $u_{\varepsilon}$ be respectively the sup and inf convolution of $u$ in $\mathbb{R}^{N}$, i.e.,

$$
\begin{aligned}
u^{\varepsilon}(x) & :=\sup _{y \in \mathbb{R}^{N}}\left(u(y)-\frac{1}{2 \varepsilon}|x-y|^{2}\right) \\
\text { and } \quad u_{\varepsilon}(x) & :=\inf _{y \in \mathbb{R}^{N}}\left(u(y)+\frac{1}{2 \varepsilon}|x-y|^{2}\right) .
\end{aligned}
$$

We notice that

$$
u^{\varepsilon}(x) \geqslant u(x) \geqslant u_{\varepsilon}(x) .
$$

Moreover, $u^{\varepsilon}$ is semiconvex and is a subsolution of 3.2 in $B_{1-\rho}$ and $u_{\varepsilon}$ is semiconcave and is a supersolution of 3.3 in $B_{1-\rho}$, for some $\rho=\rho(\varepsilon)>0$, which is infinitesimal as $\varepsilon \searrow 0$, see e.g. Proposition III.2 in 2 .

Since (3.7) does not hold true, there exists $\alpha \in(0,2 s)$ such that, for any $L>0$ and $\varepsilon>0$,

$$
\begin{aligned}
& \sup _{\left(x_{1}, x_{2}\right) \in \mathbb{R}^{2 N}} u^{\varepsilon}\left(x_{1}\right)-u_{\varepsilon}\left(x_{2}\right)-L\left|x_{1}-x_{2}\right|^{\alpha}-\psi\left(x_{1}\right) \\
\geqslant & \sup _{\left(x_{1}, x_{2}\right) \in \mathbb{R}^{2 N}} u\left(x_{1}\right)-u\left(x_{2}\right)-L\left|x_{1}-x_{2}\right|^{\alpha}-\psi\left(x_{1}\right)>0,
\end{aligned}
$$

where we also used 3.8 . Then, for any $L>0$ and $\varepsilon>0$, the supremum on $\mathbb{R}^{2 N}$ of the function

$$
u^{\varepsilon}\left(x_{1}\right)-u_{\varepsilon}\left(x_{2}\right)-L\left|x_{1}-x_{2}\right|^{\alpha}-\psi\left(x_{1}\right)
$$

is positive and is attained at some point $\left(\bar{x}_{1}, \bar{x}_{2}\right) \in \mathbb{R}^{2 N}$. Moreover, for $\varepsilon$ small enough, we have that $\bar{x}_{1} \neq \bar{x}_{2}$. We remark that

$$
\left|\bar{x}_{1}-\bar{x}_{2}\right| \leqslant\left(\frac{2\|u\|_{L^{\infty}\left(\mathbb{R}^{N}\right)}}{L}\right)^{\frac{1}{\alpha}} .
$$

Using that $\phi \geqslant 1$ in $\mathbb{R}^{N} \backslash B_{3 / 4}$ and (3.6), we see that, for all $x_{1} \in \mathbb{R}^{N} \backslash B_{3 / 4}$,

$$
u^{\varepsilon}\left(x_{1}\right)-u_{\varepsilon}\left(x_{2}\right)-L\left|x_{1}-x_{2}\right|^{\alpha}-\psi\left(x_{1}\right) \leqslant u^{\varepsilon}\left(x_{1}\right)-u_{\varepsilon}\left(x_{2}\right)-2\|u\|_{L^{\infty}\left(\mathbb{R}^{N}\right)} \leqslant o_{\varepsilon}(1),
$$

where $o_{\varepsilon}(1) \rightarrow 0$ as $\varepsilon \searrow 0$. Thus we must have $x_{1} \in B_{3 / 4}$ for $\varepsilon$ small enough, and by (3.10), if

$$
L \geqslant 16\|u\|_{L^{\infty}\left(\mathbb{R}^{N}\right)},
$$

we have that

$$
\bar{x}_{1}, \bar{x}_{2} \in B_{7 / 8}
$$


The function in $(3.9)$ is semiconvex, hence, by Aleksandrov's Theorem, twice differentiable almost everywhere. Let us now introduce a perturbation of it, for which we can choose maximum points of twice differentiability.

First we transform $\left(\bar{x}_{1}, \bar{x}_{2}\right)$ into a strict maximum point. In order to do that, we consider a smooth function $h: \mathbb{R}^{+} \rightarrow \mathbb{R}$, with compact support, such that $h(0)=0$ and $h(t)>0$ for $0<t<1$, we fix a small $\beta>0$ and we set

$$
\theta\left(x_{1}, x_{2}\right):=\beta h\left(\left|x_{1}-\bar{x}_{1}\right|^{2}\right)+\beta h\left(\left|x_{2}-\bar{x}_{2}\right|^{2}\right) .
$$

Clearly, $\left(\bar{x}_{1}, \bar{x}_{2}\right)$ is a strict maximum point of $u^{\varepsilon}\left(x_{1}\right)-u_{\varepsilon}\left(x_{2}\right)-L\left|x_{1}-x_{2}\right|^{\alpha}-\psi\left(x_{1}\right)-$ $\theta\left(x_{1}, x_{2}\right)$.

Next we consider a smooth function $\tau: \mathbb{R}^{N} \rightarrow \mathbb{R}$ such that $\tau(x)=1$ if $|x| \leqslant 1 / 2$ and $\tau(x)=0$ for $|x| \geqslant 1$. By Jensen's Lemma, see e.g. Lemma A.3 of [18, for every small and positive $\delta$, there exist $q_{1}^{\delta}, q_{2}^{\delta} \in \mathbb{R}^{N}$ with $\left|q_{1}^{\delta}\right|,\left|q_{2}^{\delta}\right| \leqslant \delta$, such that the function

$$
\Phi\left(x_{1}, x_{2}\right):=u^{\varepsilon}\left(x_{1}\right)-u_{\varepsilon}\left(x_{2}\right)-L\left|x_{1}-x_{2}\right|^{\alpha}-\varphi_{1}\left(x_{1}\right)-\varphi_{2}\left(x_{2}\right),
$$

where

$$
\begin{aligned}
\varphi_{1}\left(x_{1}\right) & :=\psi\left(x_{1}\right)+\beta h\left(\left|x_{1}-\bar{x}_{1}\right|^{2}\right)+\tau\left(x_{1}-\bar{x}_{1}\right) q_{1}^{\delta} \cdot x_{1}, \\
\text { and } \quad \varphi_{2}\left(x_{2}\right) & :=\beta h\left(\left|x_{2}-\bar{x}_{2}\right|^{2}\right)+\tau\left(x_{2}-\bar{x}_{2}\right) q_{2}^{\delta} \cdot x_{2},
\end{aligned}
$$

has a maximum at $\left(x_{1}^{\delta}, x_{2}^{\delta}\right)$, with

$$
\left|x_{1}^{\delta}-\bar{x}_{1}\right|,\left|x_{2}^{\delta}-\bar{x}_{2}\right| \leqslant \delta
$$

and $u^{\varepsilon}\left(x_{1}\right)-u_{\varepsilon}\left(x_{2}\right)$ is twice differentiable at $\left(x_{1}^{\delta}, x_{2}^{\delta}\right)$. In particular, $u^{\varepsilon}$ is twice differentiable with respect to $x_{1}$ at $x_{1}^{\delta}$ and $u_{\varepsilon}$ is twice differentiable with respect to $x_{2}$ at $x_{2}^{\delta}$.

We remark that the function $\tau$ has been introduced to make $\mathscr{L}^{2, r} \varphi_{1}$ and $\mathscr{L}^{2, r} \varphi_{2}$ finite. Also, for $\delta$ small enough, and $\rho$ small enough, by (3.11) and 3.13), we have that

$$
x_{1}^{\delta}, x_{2}^{\delta} \in B_{1-\rho},
$$

and that $x_{1}^{\delta} \neq x_{2}^{\delta}$. In particular, this will allow us to compute the derivatives of the function in (3.12).

Since $\left(x_{1}^{\delta}, x_{2}^{\delta}\right)$ is a maximum point for $\Phi$, we have

$$
\begin{aligned}
& \nabla u^{\varepsilon}\left(x_{1}^{\delta}\right)=\nabla \varphi_{1}\left(x_{1}^{\delta}\right)+\alpha L\left|x_{1}^{\delta}-x_{2}^{\delta}\right|^{\alpha-2}\left(x_{1}^{\delta}-x_{2}^{\delta}\right) \\
& \text { and } \nabla u_{\varepsilon}\left(x_{2}^{\delta}\right)=-\nabla \varphi_{2}\left(x_{2}^{\delta}\right)+\alpha L\left|x_{1}^{\delta}-x_{2}^{\delta}\right|^{\alpha-2}\left(x_{1}^{\delta}-x_{2}^{\delta}\right) \text {. }
\end{aligned}
$$

Moreover the inequalities

$$
\begin{array}{ll} 
& \Phi\left(x_{1}^{\delta}+z, x_{2}^{\delta}\right) \leqslant \Phi\left(x_{1}^{\delta}, x_{2}^{\delta}\right), \\
& \Phi\left(x_{1}^{\delta}, x_{2}^{\delta}+z\right) \leqslant \Phi\left(x_{1}^{\delta}, x_{2}^{\delta}\right) \\
\text { and } \quad & \Phi\left(x_{1}^{\delta}+z, x_{2}^{\delta}+z\right) \leqslant \Phi\left(x_{1}^{\delta}, x_{2}^{\delta}\right),
\end{array}
$$


for any $z \in \mathbb{R}^{N}$, together with 3.15 , give respectively:

$$
\begin{aligned}
& u^{\varepsilon}\left(x_{1}^{\delta}+z\right)-u^{\varepsilon}\left(x_{1}^{\delta}\right)-\nabla u^{\varepsilon}\left(x_{1}^{\delta}\right) \cdot z \\
\leqslant & \varphi_{1}\left(x_{1}^{\delta}+z\right)-\varphi_{1}\left(x_{1}^{\delta}\right)-\nabla \varphi_{1}\left(x_{1}^{\delta}\right) \cdot z \\
\quad & \quad+L\left|x_{1}^{\delta}+z-x_{2}^{\delta}\right|^{\alpha}-L\left|x_{1}^{\delta}-x_{2}^{\delta}\right|^{\alpha}-\alpha L\left|x_{1}^{\delta}-x_{2}^{\delta}\right|^{\alpha-2}\left(x_{1}^{\delta}-x_{2}^{\delta}\right) \cdot z,
\end{aligned}
$$

and

$$
\begin{aligned}
& \quad-\left(u_{\varepsilon}\left(x_{2}^{\delta}+z\right)-u_{\varepsilon}\left(x_{2}^{\delta}\right)-\nabla u_{\varepsilon}\left(x_{2}^{\delta}\right) \cdot z\right) \\
& \leqslant \varphi_{2}\left(x_{2}^{\delta}+z\right)-\varphi_{2}\left(x_{2}^{\delta}\right)-\nabla \varphi_{2}\left(x_{2}^{\delta}\right) \cdot z \\
& \quad+L\left|x_{1}^{\delta}-z-x_{2}^{\delta}\right|^{\alpha}-L\left|x_{1}^{\delta}-x_{2}^{\delta}\right|^{\alpha}+\alpha L\left|x_{1}^{\delta}-x_{2}^{\delta}\right|^{\alpha-2}\left(x_{1}^{\delta}-x_{2}^{\delta}\right) \cdot z,
\end{aligned}
$$

and, for any $r>0$,

$$
\begin{aligned}
& \quad u^{\varepsilon}\left(x_{1}^{\delta}+z\right)-u^{\varepsilon}\left(x_{1}^{\delta}\right)-\chi_{B_{r}}(z) \nabla u^{\varepsilon}\left(x_{1}^{\delta}\right) \cdot z \\
& \leqslant u_{\varepsilon}\left(x_{2}^{\delta}+z\right)-u_{\varepsilon}\left(x_{2}^{\delta}\right)-\chi_{B_{r}}(z) \nabla u_{\varepsilon}\left(x_{2}^{\delta}\right) \cdot z \\
& \quad+\varphi_{1}\left(x_{1}^{\delta}+z\right)-\varphi_{1}\left(x_{1}^{\delta}\right)-\chi_{B_{r}}(z) \nabla \varphi_{1}\left(x_{1}^{\delta}\right) \cdot z \\
& \quad+\varphi_{2}\left(x_{2}^{\delta}+z\right)-\varphi_{2}\left(x_{2}^{\delta}\right)-\chi_{B_{r}}(z) \nabla \varphi_{2}\left(x_{2}^{\delta}\right) \cdot z .
\end{aligned}
$$

The last inequality in particular implies that

$$
\mathscr{L}^{2, r} u^{\varepsilon}\left(x_{1}^{\delta}\right) \geqslant \mathscr{L}^{2, r} u_{\varepsilon}\left(x_{2}^{\delta}\right)+\mathscr{L}^{2, r} \varphi_{1}\left(x_{1}^{\delta}\right)+\mathscr{L}^{2, r} \varphi_{2}\left(x_{2}^{\delta}\right),
$$

and

$$
D^{2} u^{\varepsilon}\left(x_{1}^{\delta}\right)-D^{2} u_{\varepsilon}\left(x_{2}^{\delta}\right) \leqslant C\left(\beta+\|u\|_{L^{\infty}\left(\mathbb{R}^{N}\right)}\right) I_{N},
$$

where $I_{N}$ is the $N \times N$ identity matrix. Here and henceforth $C$ denotes various positive constants independent of the parameters.

Now, using that $u^{\varepsilon}$ and $u_{\varepsilon}$ are respectively subsolution of $(3.2)$ and supersolution of (3.3) in $B_{1-\rho}$, and recalling (3.5) and (3.14), we have that

$$
-\eta \Delta u^{\varepsilon}\left(x_{1}^{\delta}\right)+\mathscr{L}^{1, r} u^{\varepsilon}\left(x_{1}^{\delta}\right)+\mathscr{L}^{2, r} u^{\varepsilon}\left(x_{1}^{\delta}\right)+f_{1} \leqslant 0
$$

and

$$
-\eta \Delta u_{\varepsilon}\left(x_{2}^{\delta}\right)+\mathscr{L}^{1, r} u_{\varepsilon}\left(x_{2}^{\delta}\right)+\mathscr{L}^{2, r} u_{\varepsilon}\left(x_{2}^{\delta}\right)+f_{2} \geqslant 0 .
$$

Thus, by subtracting (3.22) to (3.21) and using (3.19) and (3.20), we obtain

$$
\mathscr{L}^{1, r} u^{\varepsilon}\left(x_{1}^{\delta}\right)-\mathscr{L}^{1, r} u_{\varepsilon}\left(x_{2}^{\delta}\right)+f_{1}-f_{2}-C\left(\beta+\|u\|_{L^{\infty}\left(\mathbb{R}^{N}\right)}\right) \leqslant 0 .
$$

Next, let us estimate the term $\mathscr{L}^{1, r} u^{\varepsilon}\left(x_{1}^{\delta}\right)-\mathscr{L}^{1, r} u_{\varepsilon}\left(x_{2}^{\delta}\right)$ and show that it contains a main negative part. For $0<\nu_{0}<1$, let us denote by $A_{r}$ the cone

$$
A_{r}:=\left\{z \in B_{r},\left|z \cdot\left(x_{1}^{\delta}-x_{2}^{\delta}\right)\right| \geqslant \nu_{0}|z|\left|x_{1}^{\delta}-x_{2}^{\delta}\right|\right\} .
$$

Then

$$
\begin{aligned}
& \mathscr{L}^{1, r} u^{\varepsilon}\left(x_{1}^{\delta}\right)-\mathscr{L}^{1, r} u_{\varepsilon}\left(x_{2}^{\delta}\right) \\
= & -\int_{A_{r}}\left[u^{\varepsilon}\left(x_{1}^{\delta}+z\right)-u^{\varepsilon}\left(x_{1}^{\delta}\right)-\nabla u^{\varepsilon}\left(x_{1}^{\delta}\right) \cdot z-\left(u_{\varepsilon}\left(x_{2}^{\delta}+z\right)-u_{\varepsilon}\left(x_{2}^{\delta}\right)-\nabla u_{\varepsilon}\left(x_{2}^{\delta}\right) \cdot z\right)\right] K(z) d z \\
& -\int_{B_{r} \backslash A_{r}}\left[u^{\varepsilon}\left(x_{1}^{\delta}+z\right)-u^{\varepsilon}\left(x_{1}^{\delta}\right)-\nabla u^{\varepsilon}\left(x_{1}^{\delta}\right) \cdot z-\left(u_{\varepsilon}\left(x_{2}^{\delta}+z\right)-u_{\varepsilon}\left(x_{2}^{\delta}\right)-\nabla u_{\varepsilon}\left(x_{2}^{\delta}\right) \cdot z\right)\right] K(z) d z \\
= & :-T_{1}-T_{2} .
\end{aligned}
$$


From (3.18) we have

$$
T_{2} \leqslant C\left(\beta+\|u\|_{L^{\infty}\left(\mathbb{R}^{N}\right)}\right) .
$$

Let us estimate $T_{1}$. Using (3.16) and (3.17), and successively making the change of variable $z \rightarrow-z$, we get the following estimate of $T_{1}$ :

$$
\begin{aligned}
T_{1} \leqslant \int_{A_{r}} & {\left[L\left|x_{1}^{\delta}+z-x_{2}^{\delta}\right|^{\alpha}-L\left|x_{1}^{\delta}-x_{2}^{\delta}\right|^{\alpha}-\alpha L\left|x_{1}^{\delta}-x_{2}^{\delta}\right|^{\alpha-2}\left(x_{1}^{\delta}-x_{2}^{\delta}\right) \cdot z\right] K(z) d z } \\
& +C\left(\beta+\|u\|_{L^{\infty}\left(\mathbb{R}^{N}\right)}\right) \\
& +\int_{A_{r}}\left[L\left|x_{1}^{\delta}-z-x_{2}^{\delta}\right|^{\alpha}-L\left|x_{1}^{\delta}-x_{2}^{\delta}\right|^{\alpha}+\alpha L\left|x_{1}^{\delta}-x_{2}^{\delta}\right|^{\alpha-2}\left(x_{1}^{\delta}-x_{2}^{\delta}\right) \cdot z\right] K(z) d z \\
= & 2 \int_{A_{r}}\left[L\left|x_{1}^{\delta}+z-x_{2}^{\delta}\right|^{\alpha}-L\left|x_{1}^{\delta}-x_{2}^{\delta}\right|^{\alpha}-\alpha L\left|x_{1}^{\delta}-x_{2}^{\delta}\right|^{\alpha-2}\left(x_{1}^{\delta}-x_{2}^{\delta}\right) \cdot z\right] K(z) d z \\
& +C\left(\beta+\|u\|_{L^{\infty}\left(\mathbb{R}^{N}\right)}\right) \\
\leqslant & \alpha \\
& \int_{A_{r}\{|t| \leqslant 1\}}\left\{\left|x_{1}^{\delta}-x_{2}^{\delta}+t z\right|^{\alpha-4}\left(\left|x_{1}^{\delta}-x_{2}^{\delta}+t z\right|^{2}|z|^{2}-(2-\alpha)\left[\left(x_{1}^{\delta}-x_{2}^{\delta}+t z\right) \cdot z\right]^{2}\right)\right\} K(z) d z \\
& +C\left(\beta+\|u\|_{L^{\infty}\left(\mathbb{R}^{N}\right)}\right) .
\end{aligned}
$$

Let us fix $r:=\sigma\left|x_{1}^{\delta}-x_{2}^{\delta}\right|$, for some $\sigma>0$. Then, for $z \in A_{r}$,

$$
\begin{aligned}
& \left|x_{1}^{\delta}-x_{2}^{\delta}+t z\right| \leqslant(1+\sigma)\left|x_{1}^{\delta}-x_{2}^{\delta}\right| \\
\text { and } \quad & \left|\left(x_{1}^{\delta}-x_{2}^{\delta}+t z\right) \cdot z\right| \geqslant\left|\left(x_{1}^{\delta}-x_{2}^{\delta}\right) \cdot z\right|-|z|^{2} \geqslant\left(\nu_{0}-\sigma\right)\left|x_{1}^{\delta}-x_{2}^{\delta}\right||z| .
\end{aligned}
$$

Let us choose $0<\sigma<\nu_{0}<1$ such that

$$
C_{0}:=-(1+\sigma)^{2}+(2-\alpha)\left(\nu_{0}-\sigma\right)^{2}>0,
$$

then by (1.4),

$$
\begin{aligned}
T_{1} & \leqslant-C C_{0} L\left|x_{1}^{\delta}-x_{2}^{\delta}\right|^{\alpha-2} \int_{A_{r}}|z|^{2} K(z) d z+C\left(\beta+\|u\|_{L^{\infty}\left(\mathbb{R}^{N}\right)}\right) \\
& \leqslant-C C_{0} L\left|x_{1}^{\delta}-x_{2}^{\delta}\right|^{\alpha-2} r^{2-2 s}+C\left(\beta+\|u\|_{L^{\infty}\left(\mathbb{R}^{N}\right)}\right) \\
& \leqslant-C C_{0} L\left|x_{1}^{\delta}-x_{2}^{\delta}\right|^{\alpha-2 s}+C\left(\beta+\|u\|_{L^{\infty}\left(\mathbb{R}^{N}\right)}\right) .
\end{aligned}
$$

From (3.23), 3.24, 3.25) and (3.26), we obtain

$$
C C_{0} L\left|x_{1}^{\delta}-x_{2}^{\delta}\right|^{\alpha-2 s}-C\left(\beta+\|u\|_{L^{\infty}\left(\mathbb{R}^{N}\right)}\right)+f_{1}-f_{2} \leqslant 0 .
$$

Letting $\delta$ go to 0 , the last inequality and 3.13 yield

$$
C C_{0} L\left|\bar{x}_{1}-\bar{x}_{2}\right|^{\alpha-2 s} \leqslant C\left(\beta+\|u\|_{L^{\infty}\left(\mathbb{R}^{N}\right)}\right)+f_{2}-f_{1} .
$$

Thus, since $\alpha-2 s<0$, using (3.10) and letting $\beta$ go to 0 , we finally obtain

$$
L \leqslant C\left(f_{2}-f_{1}+\|u\|_{L^{\infty}\left(\mathbb{R}^{N}\right)}\right)^{\frac{\alpha}{2 s}}\|u\|_{L^{\infty}\left(\mathbb{R}^{N}\right)}^{1-\frac{\alpha}{2 s}} .
$$

Since $L$ was chosen as big as we wish, we get a contradiction for

$$
L>C\left(f_{2}-f_{1}+\|u\|_{L^{\infty}\left(\mathbb{R}^{N}\right)}\right)^{\frac{\alpha}{2 s}}\|u\|_{L^{\infty}\left(\mathbb{R}^{N}\right)}^{1-\frac{\alpha}{2 s}} .
$$

This proves 3.4 . 
With the aid of Lemma 3.1. we can prove the following regularity result (with uniform bounds):

Lemma 3.2. Let $T>1, \eta, \mu \in(0,1), \rho>0, \zeta \in \mathscr{Z}$. Let $Q \in L^{\infty}(\mathbb{R})$ be a solution of

$-\eta \ddot{Q}(x)+\mu\left(Q-Q_{\zeta_{1}, \zeta_{2}}^{\sharp}\right)+\mathscr{L}(Q)(x)+a(x) W^{\prime}(Q(x))=0, \quad$ for any $x \in(-4 T, 4 T)$.

Suppose that

$$
Q(x) \in \overline{B_{\rho}(\zeta)} \text { for any } x \in(-4 T, 4 T)
$$

Then, for any $\alpha \in(0,2 s)$,

$$
[Q]_{C^{0, \alpha}(-T, T)} \leqslant C T^{-\alpha}\left(\|Q\|_{L^{\infty}(\mathbb{R})}+T^{2 s} \rho+T^{2 s} \mu\right)^{\frac{\alpha}{2 s}} \rho^{1-\frac{\alpha}{2 s}},
$$

for some $C>0$ independent of $\eta$ and depending on structural constants.

Proof. Up to a translation, we assume that $\zeta=0$, hence 3.27$)$ becomes

$$
|Q(x)| \leqslant \rho, \text { for any } x \in(-4 T, 4 T) .
$$

We let $\tau_{o} \in C_{0}^{\infty}([-4,4],[0,1])$ be such that $\tau_{o}(x)=1$ for any $x \in[-3,3]$. We define $\tau(x):=\tau_{o}(x / T)$ and $u(x):=\tau(x) Q(x)$. Notice that, by (3.29),

$$
|u(x)| \leqslant \rho \text { for any } x \in \mathbb{R} .
$$

Arguing as in Lemma 4.1 in 21], we see that $u$ is solution of

$$
-\eta \ddot{u}+\mathscr{L}(u)=f \quad \text { in }(-2 T, 2 T),
$$

for some function $f$ satisfying

$$
\|f\|_{L^{\infty}(-2 T, 2 T)} \leqslant \frac{C\|Q\|_{L^{\infty}(\mathbb{R})}}{T^{2 s}}+C \rho+C \mu,
$$

with $C>0$ independent of $\eta$. Let $v(x):=u(T x)$, then $v$ is a solution of

$$
-\eta T^{2(s-1)} \ddot{v}+\mathscr{L}(v)=T^{2 s} f \quad \text { in }(-2,2) .
$$

Therefore, by Lemma 3.1 and 3.30 , we have that, for any $\alpha \in(0,2 s)$,

$$
[v]_{C^{0, \alpha}(-1,1)} \leqslant C\left(\|Q\|_{L^{\infty}(\mathbb{R})}+T^{2 s} \rho+T^{2 s} \mu\right)^{\frac{\alpha}{2 s}} \rho^{1-\frac{\alpha}{2 s}} .
$$

Scaling back we get 3.28 . 


\section{Energy estimates}

The goal of this section is to provide suitable integral estimates, with the aim of bounding the energy from below (this bound is crucial to apply minimization methods in the variational arguments exploited in the forthcoming Lemma 5.1). More precisely, we provide a bound on the "mixed term" of the energy functional, as defined in 2.1). We remark that this is somehow an "unpleasant" term to consider, since, differently from the classical case, it cannot be reabsorbed into the quadratic terms in the energy by the Cauchy-Schwarz inequality, since it would produce infinite contributions when arguing in this way.

Lemma 4.1. Let $v \in L^{\infty}(\mathbb{R})$. Then

$$
\left|\mathscr{B}_{\mathbb{R}, \mathbb{R}}\left(v, Q_{\zeta_{1}, \zeta_{2}}^{\sharp}\right)\right| \leqslant \text { const }\left\|Q_{\zeta_{1}, \zeta_{2}}^{\sharp}\right\|_{C^{1}(\mathbb{R})}\left([v]_{K, \mathbb{R} \times \mathbb{R}}+\|v\|_{L^{2}(\mathbb{R})}\right) .
$$

Proof. We set $I_{-}:=(-\infty,-2), I_{+}:=(2,+\infty)$ and $J:=[-2,2]$, and we notice that $\mathscr{B}_{I_{-}, I_{-}}\left(v, Q_{\zeta_{1}, \zeta_{2}}^{\sharp}\right)=\mathscr{B}_{I_{+}, I_{+}}\left(v, Q_{\zeta_{1}, \zeta_{2}}^{\sharp}\right)=0$, since $Q_{\zeta_{1}, \zeta_{2}}^{\sharp}$ is constant on $I_{-} \cup I_{+}$. Using this and $(2.3)$, we see that

$$
\begin{aligned}
\mathscr{B}_{\mathbb{R}, \mathbb{R}}\left(v, Q_{\zeta_{1}, \zeta_{2}}^{\sharp}\right) & =\mathscr{B}_{J, J}\left(v, Q_{\zeta_{1}, \zeta_{2}}^{\sharp}\right)+2 \mathscr{B}_{J, I_{-}}\left(v, Q_{\zeta_{1}, \zeta_{2}}^{\sharp}\right) \\
& +2 \mathscr{B}_{I_{-}, I_{+}}\left(v, Q_{\zeta_{1}, \zeta_{2}}^{\sharp}\right)+2 \mathscr{B}_{J, I_{+}}\left(v, Q_{\zeta_{1}, \zeta_{2}}^{\sharp}\right) .
\end{aligned}
$$

Moreover, if $x \in[-2,1]$ and $y \in(2,+\infty)$ we have that

$$
|x-y|=y-x \geqslant \frac{y}{2}+1-1=\frac{y}{2},
$$

and hence, recalling 1.4 , we have that

$$
\begin{aligned}
& \iint_{[-2,1] \times(2,+\infty)}\left|Q_{\zeta_{1}, \zeta_{2}}^{\sharp}(x)-\zeta_{2}\right|^{2} K(x-y) d x d y \\
\leqslant & \text { const }\left\|Q_{\zeta_{1}, \zeta_{2}}^{\sharp}\right\|_{L^{\infty}(\mathbb{R})}^{2} \iint_{[-2,1] \times(2,+\infty)} y^{-1-2 s} d x d y \\
\leqslant & \text { const }\left\|Q_{\zeta_{1}, \zeta_{2}}^{\sharp}\right\|_{L^{\infty}(\mathbb{R})}^{2} .
\end{aligned}
$$

Therefore, by the Cauchy-Schwarz inequality we find that

$$
\begin{aligned}
& \left|\mathscr{B}_{J, I_{+}}\left(v, Q_{\zeta_{1}, \zeta_{2}}^{\sharp}\right)\right| \\
= & \left|\iint_{[-2,2] \times(2,+\infty)}(v(x)-v(y))\left(Q_{\zeta_{1}, \zeta_{2}}^{\sharp}(x)-\zeta_{2}\right) K(x-y) d x d y\right| \\
\leqslant & \sqrt{\iint_{[-2,1] \times(2,+\infty)}|v(x)-v(y)|^{2} K(x-y) d x d y} \\
& \times \sqrt{\iint_{[-2,1] \times(2,+\infty)}\left|Q_{\zeta_{1}, \zeta_{2}}^{\sharp}(x)-\zeta_{2}\right|^{2} K_{(x-y) d x d y}} \\
\leqslant & \text { const }\left\|Q_{\zeta_{1}, \zeta_{2}}^{\sharp}\right\|_{L^{\infty}(\mathbb{R})}[v]_{K, \mathbb{R} \times \mathbb{R}} .
\end{aligned}
$$


Similarly, we see that

$$
\left|\mathscr{B}_{J, I_{-}}\left(v, Q_{\zeta_{1}, \zeta_{2}}^{\sharp}\right)\right| \leqslant \text { const }\left\|Q_{\zeta_{1}, \zeta_{2}}^{\sharp}\right\|_{L^{\infty}(\mathbb{R})}[v]_{K, \mathbb{R} \times \mathbb{R}} .
$$

Also, we have that

$$
\begin{aligned}
& \left|\mathscr{B}_{I_{-}, I_{+}}\left(v, Q_{\zeta_{1}, \zeta_{2}}^{\sharp}\right)\right|=\left|\iint_{(-\infty,-2) \times(2,+\infty)}(v(x)-v(y))\left(\zeta_{1}-\zeta_{2}\right) K(x-y) d x d y\right| \\
& \leqslant \text { const }\left\|Q_{\zeta_{1}, \zeta_{2}}^{\sharp}\right\|_{L^{\infty}(\mathbb{R})} \iint_{(-\infty,-2) \times(2,+\infty)}(|v(x)|+|v(y)|)(y-x)^{-1-2 s} d x d y \\
& \leqslant \text { const }\left\|Q_{\zeta_{1}, \zeta_{2}}^{\sharp}\right\|_{L^{\infty}(\mathbb{R})}\left[\int_{(-\infty,-2)} \frac{|v(x)|}{(2-x)^{2 s}} d x+\int_{(2,+\infty)} \frac{|v(y)|}{(y+2)^{2 s}} d y\right] .
\end{aligned}
$$

In addition, using the Cauchy-Schwarz inequality we see that

$$
\begin{aligned}
\int_{(2,+\infty)} \frac{|v(y)|}{(y+2)^{2 s}} d y & \leqslant \sqrt{\int_{\mathbb{R}}|v(y)|^{2} d y \int_{\mathbb{R}} \frac{d y}{(y+2)^{4 s}}} \\
& \leqslant \text { const } \sqrt{\int_{\mathbb{R}}|v(x)|^{2} d x .}
\end{aligned}
$$

We stress that we have used condition (1.5) here. Similarly,

$$
\int_{(-\infty,-2)} \frac{|v(x)|}{(2-x)^{2 s}} d x \leqslant \text { const } \sqrt{\int_{\mathbb{R}}|v(x)|^{2} d x} .
$$

Plugging this and 4.5) into 4.4, we thus conclude that

$$
\left|\mathscr{B}_{I_{-}, I_{+}}\left(v, Q_{\zeta_{1}, \zeta_{2}}^{\sharp}\right)\right| \leqslant \text { const }\left\|Q_{\zeta_{1}, \zeta_{2}}^{\sharp}\right\|_{L^{\infty}(\mathbb{R})} \sqrt{\int_{\mathbb{R}}|v(x)|^{2} d x} .
$$

Furthermore, by the Cauchy-Schwarz inequality and 2.4, we have that

$$
\begin{aligned}
& \left|\mathscr{B}_{J, J}\left(v, Q_{\zeta_{1}, \zeta_{2}}^{\sharp}\right)\right| \\
\leqslant & \sqrt{\iint_{J \times J}|v(x)-v(y)|^{2} K(x-y) d x d y \iint_{J \times J}\left|\left(Q_{\zeta_{1}, \zeta_{2}}^{\sharp}\right)(x)-Q_{\zeta_{1}, \zeta_{2}}^{\sharp}(y)\right|^{2} K(x-y) d x d y} \\
\leqslant & \operatorname{const}[v]_{K, \mathbb{R} \times \mathbb{R}} \sqrt{\iint_{J \times J}\left|\left(Q_{\zeta_{1}, \zeta_{2}}^{\sharp}\right)(x)-Q_{\zeta_{1}, \zeta_{2}}^{\sharp}(y)\right|^{2} K(x-y) d x d y .}
\end{aligned}
$$

Now, using that $Q_{\zeta_{1}, \zeta_{2}}^{\sharp}(x)=\zeta_{1}$ for any $x \in(-\infty,-1)$ and $Q_{\zeta_{1}, \zeta_{2}}^{\sharp}(x)=\zeta_{2}$ for any $x \in(1,+\infty)$, we have that

$$
\begin{aligned}
& \iint_{J \times J}\left|\left(Q_{\zeta_{1}, \zeta_{2}}^{\sharp}\right)(x)-Q_{\zeta_{1}, \zeta_{2}}^{\sharp}(y)\right|^{2} K(x-y) d x d y \\
& \quad=\int_{-2}^{2} \int_{-2}^{2}\left|\left(Q_{\zeta_{1}, \zeta_{2}}^{\sharp}\right)(x)-Q_{\zeta_{1}, \zeta_{2}}^{\sharp}(y)\right|^{2} K(x-y) d x d y \leqslant \text { const }\left\|Q_{\zeta_{1}, \zeta_{2}}^{\sharp}\right\|_{C^{1}(\mathbb{R})}^{2} .
\end{aligned}
$$


From this and (4.7), we find that

$$
\left|\mathscr{B}_{J, J}\left(v, Q_{\zeta_{1}, \zeta_{2}}^{\sharp}\right)\right| \leqslant \text { const }[v]_{K, \mathbb{R} \times \mathbb{R}}\left\|Q_{\zeta_{1}, \zeta_{2}}^{\sharp}\right\|_{C^{1}(\mathbb{R})} .
$$

Combining this with 4.1), 4.2), 4.3) and (4.6), we obtain the desired result.

\section{Variational methods and constrained minimization for a perturbed problem}

Fixed $\zeta_{1}, \zeta_{2} \in \mathscr{Z}$ and $r \in\left(0, \min \left\{\delta_{0}, r_{0}\right\}\right]$ (where $r_{0}$ and $\delta_{0}$ are given by 1.4 and (1.8), respectively), we construct constrained minimizers for our variational problems. To this aim, we take $b_{1} \leqslant-1$ and $b_{2} \geqslant 1$ and consider $\phi$ and $\psi$ solutions to

$$
\begin{cases}-\eta \ddot{\phi}+\mathscr{L} \phi=C_{0} & \text { in }\left(b_{1}-\tau, b_{2}+\tau\right), \\ \phi=\zeta_{1}+r & \text { in }\left(-\infty, b_{1}-\tau\right], \\ \phi=\zeta_{2}+r & \text { in }\left[b_{2}+\tau,+\infty\right),\end{cases}
$$

and

$$
\begin{cases}-\eta \ddot{\psi}+\mathscr{L} \psi=-C_{0} & \text { in }\left(b_{1}-\tau, b_{2}+\tau\right) \\ \psi=\zeta_{1}-r & \text { in }\left(-\infty, b_{1}-\tau\right] \\ \psi=\zeta_{2}-r & \text { in }\left[b_{2}+\tau,+\infty\right)\end{cases}
$$

where

$$
C_{0}:=\left\|a W^{\prime}\right\|_{L^{\infty}(\mathbb{R})}+2\left|\zeta_{1}\right|+2\left|\zeta_{2}\right|+1
$$

and $\tau \in(0,1)$. It is known that solutions to (5.1) and (5.2) with $\eta=0$ grow like $d^{s}(x)$ plus the boundary data away from the boundary of $\left(b_{1}-\tau, b_{2}+\tau\right)$, where $d(x)$ is the distance function to the boundary of $\left(b_{1}-\tau, b_{2}+\tau\right)$, see 37. Thus, by stability of viscosity solutions, there exist $c, C>0$ such that, for $\tau$ small enough,

$$
\begin{cases}c\left(x-b_{1}+\tau\right)^{s}+o_{\eta}(1) \leqslant \phi(x)-\zeta_{1}-r \leqslant C\left(x-b_{1}+\tau\right)^{s}+o_{\eta}(1) & \text { for } x \in\left[b_{1}-\tau, b_{1}\right], \\ c\left(b_{2}+\tau-x\right)^{s}+o_{\eta}(1) \leqslant \phi(x)-\zeta_{2}-r \leqslant C\left(b_{2}+\tau-x\right)^{s}+o_{\eta}(1) & \text { for } x \in\left[b_{2}, b_{2}+\tau\right], \\ -C\left(x-b_{1}+\tau\right)^{s}+o_{\eta}(1) \leqslant \psi(x)-\zeta_{1}+r \leqslant-c\left(x-b_{1}+\tau\right)^{s}+o_{\eta}(1) & \text { for } x \in\left[b_{1}-\tau, b_{1}\right], \\ -C\left(b_{2}+\tau-x\right)^{s}+o_{\eta}(1) \leqslant \psi(x)-\zeta_{2}+r \leqslant-c\left(b_{2}+\tau-x\right)^{s}+o_{\eta}(1) & \text { for } x \in\left[b_{2}, b_{2}+\tau\right],\end{cases}
$$

where $o_{\eta}(1) \rightarrow 0$ as $\eta \searrow 0$. In particular, for small $\tau$,

$$
\begin{cases}\left|\phi(x)-\zeta_{1}-r\right| \leqslant \frac{r}{4} & \text { for } x \in\left[b_{1}-\tau, b_{1}\right], \\ \left|\phi(x)-\zeta_{2}-r\right| \leqslant \frac{r}{4} & \text { for } x \in\left[b_{2}, b_{2}+\tau\right], \\ \left|\psi(x)-\zeta_{1}+r\right| \leqslant \frac{r}{4} & \text { for } x \in\left[b_{1}-\tau, b_{1}\right], \\ \left|\psi(x)-\zeta_{2}+r\right| \leqslant \frac{r}{4} & \text { for } x \in\left[b_{2}, b_{2}+\tau\right] .\end{cases}
$$


Next, consider smooth functions $\Phi: \mathbb{R} \rightarrow \mathbb{R}$ and $\Psi: \mathbb{R} \rightarrow \mathbb{R}$ such that

$$
\begin{cases}\Phi(x)=\phi(x) & \text { for } x \in\left(-\infty, b_{1}-2 \tau\right] \cup\left[b_{2}+2 \tau,+\infty\right), \\ \zeta_{1}+\frac{3}{4} r \leqslant \Phi(x) \leqslant \phi(x) \leqslant \zeta_{1}+\frac{5}{4} r & \text { for } x \in\left(b_{1}-2 \tau, b_{1}\right], \\ \Phi(x) \geqslant \phi(x) & \text { for } x \in\left(b_{1}, b_{2}\right), \\ \zeta_{2}+\frac{3}{4} r \leqslant \Phi(x) \leqslant \phi(x) \leqslant \zeta_{2}+\frac{5}{4} r & \text { for }\left[b_{2}, b_{2}+2 \tau\right)\end{cases}
$$

and

$$
\begin{cases}\Psi(x)=\psi(x) & \text { for all } x \in\left(-\infty, b_{1}-2 \tau\right] \cup\left[b_{2}+2 \tau,+\infty\right) \\ \zeta_{1}-\frac{5}{4} r \leqslant \psi(x) \leqslant \Psi(x) \leqslant \zeta_{1}-\frac{3}{4} r & \text { for all } x \in\left(b_{1}-2 \tau, b_{1}\right] \\ \Psi(x) \leqslant \psi(x) & \text { for all } x \in\left(b_{1}, b_{2}\right) \\ \zeta_{2}-\frac{5}{4} r \leqslant \psi(x) \leqslant \Psi(x) \leqslant \zeta_{2}-\frac{3}{4} r & \text { for all }\left[b_{2}, b_{2}+2 \tau\right)\end{cases}
$$

With this, we can define the set

$$
\begin{aligned}
\Gamma\left(b_{1}, b_{2}\right):= & \left\{Q: \mathbb{R} \rightarrow \mathbb{R} \text { s.t. } Q-Q_{\zeta_{1}, \zeta_{2}}^{\sharp} \in H^{1}(\mathbb{R}),\right. \\
& \left.\Psi(x) \leqslant Q(x) \leqslant \Phi(x) \text { for all } x \in\left(-\infty, b_{1}\right] \cup\left[b_{2},+\infty\right)\right\} .
\end{aligned}
$$

Given $\eta, \mu \in(0,1]$, we also consider the energy functional

$$
\begin{aligned}
I_{\eta, \mu}(Q) & :=\frac{\eta}{2} \int_{\mathbb{R}}|\dot{Q}(x)|^{2} d x+\frac{\mu}{2} \int_{\mathbb{R}}\left|Q(x)-Q_{\zeta_{1}, \zeta_{2}}^{\sharp}(x)\right|^{2} d x+\int_{\mathbb{R}} a(x) W(Q(x)) d x \\
& +\frac{1}{4} \iint_{\mathbb{R} \times \mathbb{R}}\left(|Q(x)-Q(y)|^{2}-\left|Q_{\zeta_{1}, \zeta_{2}}^{\sharp}(x)-Q_{\zeta_{1}, \zeta_{2}}^{\sharp}(y)\right|^{2}\right) K(x-y) d x d y .
\end{aligned}
$$

Then, we can construct a constrained minimizer for $I_{\eta, \mu}$ in $\Gamma\left(b_{1}, b_{2}\right)$ (later on, in Proposition 10.1, we will take $b_{1}$ and $b_{2}$ conveniently separated, in order to employ condition 1.12, so to obtain an unconstrained minimizer, and then, in Section 11 . we will send $\eta \searrow 0$ for a fixed $\mu>0$. Finally, in Section 13 , we will send $\mu \searrow 0$, in order to obtain a solution of our original equation, as claimed in Theorem 1.1.

Lemma 5.1. There exists $Q_{\eta, \mu} \in \Gamma\left(b_{1}, b_{2}\right)$ such that

$$
I_{\eta, \mu}\left(Q_{\eta, \mu}\right) \leqslant I_{\eta, \mu}(Q) \text { for all } Q \in \Gamma\left(b_{1}, b_{2}\right)
$$




$$
\begin{aligned}
& \text { Also, setting } v_{\eta, \mu}:=Q_{\eta, \mu}-Q_{\zeta_{1}, \zeta_{2}}^{\sharp} \text {, we have that } \\
& {\left[v_{\eta, \mu}\right]_{H^{1}(\mathbb{R})} \leqslant \frac{\kappa}{\sqrt{\eta \mu}}} \\
& {\left[v_{\eta, \mu}\right]_{K, \mathbb{R} \times \mathbb{R}} \leqslant \frac{\kappa}{\sqrt{\mu}},} \\
& \left\|v_{\eta, \mu}\right\|_{L^{\infty}(\mathbb{R})} \leqslant \kappa, \\
& \left\|v_{\eta, \mu}\right\|_{L^{2}(\mathbb{R})} \leqslant \frac{\kappa}{\mu}, \\
& {\left[v_{\eta, \mu}\right]_{C^{0, \frac{1}{2}(\mathbb{R})}} \leqslant \frac{\kappa}{\sqrt{\eta \mu}},} \\
& \min \left\{\zeta_{1}, \zeta_{2}\right\} \leqslant Q_{\eta, \mu}(x) \leqslant \max \left\{\zeta_{1}, \zeta_{2}\right\} \quad \text { for all } x \in \mathbb{R} \\
& \text { and } E_{\mathbb{R}^{2}}\left(Q_{\eta, \mu}\right) \geqslant-\frac{\kappa}{\mu^{2}} \text {, }
\end{aligned}
$$

for some $\kappa>0$, which possibly depends on $Q_{\zeta_{1}, \zeta_{2}}^{\sharp}$ and on structural constants.

Proof. We take a minimizing sequence $Q_{j} \in \Gamma\left(b_{1}, b_{2}\right)$ for the functional $I_{\eta, \mu}$, and we let $v_{j}:=Q_{j}-Q_{\zeta_{1}, \zeta_{2}}^{\sharp} \in H^{1}(\mathbb{R})$. Defining

$$
Q_{j}^{\star}(x):= \begin{cases}Q_{j}(x) & \text { if } Q_{j}(x) \in\left(\min \left\{\zeta_{1}, \zeta_{2}\right\}, \max \left\{\zeta_{1}, \zeta_{2}\right\}\right), \\ \min \left\{\zeta_{1}, \zeta_{2}\right\} & \text { if } Q_{j}(x) \leqslant \min \left\{\zeta_{1}, \zeta_{2}\right\}, \\ \max \left\{\zeta_{1}, \zeta_{2}\right\} & \text { if } Q_{j}(x) \geqslant \max \left\{\zeta_{1}, \zeta_{2}\right\},\end{cases}
$$

we see by direct inspection that

$$
\left|Q_{j}^{\star}(x)-Q_{j}^{\star}(y)\right| \leqslant\left|Q_{j}(x)-Q_{j}(y)\right| \quad \text { and } \quad\left|\dot{Q}_{j}^{\star}(x)\right| \leqslant\left|\dot{Q}_{j}(x)\right| .
$$

Also, we point out that $W\left(Q_{j}^{\star}(x)\right)=0 \leqslant W(Q(x))$ for every $x \in\left\{Q_{j}^{\star} \neq Q_{j}\right\}$ and, as a result,

$$
W\left(Q_{j}^{\star}(x)\right) \leqslant W\left(Q_{j}(x)\right) .
$$

Moreover, in light of 1.15 , if $Q_{j}(x) \leqslant \min \left\{\zeta_{1}, \zeta_{2}\right\}$ then

$$
\begin{aligned}
& \left|Q_{j}^{\star}(x)-Q_{\zeta_{1}, \zeta_{2}}^{\sharp}(x)\right|=\left|\min \left\{\zeta_{1}, \zeta_{2}\right\}-Q_{\zeta_{1}, \zeta_{2}}^{\sharp}(x)\right|=Q_{\zeta_{1}, \zeta_{2}}^{\sharp}(x)-\min \left\{\zeta_{1}, \zeta_{2}\right\} \\
& \quad \leqslant Q_{\zeta_{1}, \zeta_{2}}^{\sharp}(x)-Q_{j}(x) \leqslant\left|Q_{j}(x)-Q_{\zeta_{1}, \zeta_{2}}^{\sharp}(x)\right|,
\end{aligned}
$$

and a similar estimate holds if $Q_{j}(x) \geqslant \max \left\{\zeta_{1}, \zeta_{2}\right\}$.

This gives that $\left|Q_{j}^{\star}(x)-Q_{\zeta_{1}, \zeta_{2}}^{\sharp}(x)\right| \leqslant\left|Q_{j}(x)-Q_{\zeta_{1}, \zeta_{2}}^{\sharp}(x)\right|$ for all $x \in \mathbb{R}$. Consequently, by 5.17) and (5.18), we see that $I_{\eta, \mu}\left(Q_{j}^{\star}\right) \leqslant I_{\eta, \mu}\left(Q_{j}\right)$.

For that reason, from now on, possibly replacing $Q_{j}$ with $Q_{j}^{\star}$, we can suppose that

$$
\min \left\{\zeta_{1}, \zeta_{2}\right\} \leqslant Q_{j}(x) \leqslant \max \left\{\zeta_{1}, \zeta_{2}\right\} \quad \text { for all } x \in \mathbb{R},
$$

and therefore

$$
\left|v_{j}(x)\right| \leqslant \kappa \quad \text { for all } x \in \mathbb{R},
$$


for some $\kappa>0$.

We also define $J_{\eta, \mu}(v):=I_{\eta, \mu}\left(Q_{\zeta_{1}, \zeta_{2}}^{\sharp}+v\right)$. In this way, the sequence $v_{j}$ is minimizing for $J_{\eta, \mu}$, and

$$
\begin{aligned}
J_{\eta, \mu}(v)= & \frac{\eta}{2} \int_{\mathbb{R}}\left(\left|\dot{Q}_{\zeta_{1}, \zeta_{2}}^{\sharp}(x)\right|^{2}+|\dot{v}(x)|^{2}+2 \dot{Q}_{\zeta_{1}, \zeta_{2}}^{\sharp}(x) \dot{v}(x)\right) d x+\frac{\mu}{2} \int_{\mathbb{R}}|v(x)|^{2} d x \\
& +\int_{\mathbb{R}} a(x) W\left(Q_{\zeta_{1}, \zeta_{2}}^{\sharp}(x)+v(x)\right) d x \\
& +\frac{1}{4} \iint_{\mathbb{R} \times \mathbb{R}}|v(x)-v(y)|^{2} K(x-y) d x d y+\frac{1}{2} \mathscr{B}_{\mathbb{R}, \mathbb{R}}\left(v, Q_{\zeta_{1}, \zeta_{2}}^{\sharp}\right) .
\end{aligned}
$$

Since $v_{j}$ is minimizing and the zero function is an admissible competitor for $J_{\eta, \mu}$, we can also suppose that

$$
J_{\eta, \mu}\left(v_{j}\right) \leqslant J_{\eta, \mu}(0)+1 \leqslant \frac{1}{2} \int_{\mathbb{R}}\left|\dot{Q}_{\zeta_{1}, \zeta_{2}}^{\sharp}(x)\right|^{2} d x+\int_{\mathbb{R}} \bar{a} W\left(Q_{\zeta_{1}, \zeta_{2}}^{\sharp}(x)\right) d x+1 \leqslant \kappa .
$$

In addition, by Cauchy-Schwarz inequality,

$$
2\left|\dot{Q}_{\zeta_{1}, \zeta_{2}}^{\sharp}(x) \cdot \dot{v}_{j}(x)\right| \leqslant 4\left|\dot{Q}_{\zeta_{1}, \zeta_{2}}^{\sharp}(x)\right|^{2}+\frac{1}{4}\left|\dot{v}_{j}(x)\right|^{2} .
$$

Combining this estimate with formulas 5.21 and 5.22 , we conclude that

$$
\begin{gathered}
\frac{3 \eta}{8} \int_{\mathbb{R}}\left|\dot{v}_{j}(x)\right|^{2} d x+\frac{\mu}{2} \int_{\mathbb{R}}\left|v_{j}(x)\right|^{2} d x+\frac{1}{4} \iint_{\mathbb{R} \times \mathbb{R}}\left|v_{j}(x)-v_{j}(y)\right|^{2} K(x-y) d x d y \\
+\frac{1}{2} \mathscr{B}_{\mathbb{R}, \mathbb{R}}\left(v_{j}, Q_{\zeta_{1}, \zeta_{2}}^{\sharp}\right) \leqslant \kappa,
\end{gathered}
$$

up to the freedom of renaming $\kappa$.

Furthermore, recalling Lemma 4.1. fixing a small additional parameter $\varepsilon>0$, and using the Cauchy-Schwarz inequality,

$$
\left|\mathscr{B}_{\mathbb{R}, \mathbb{R}}\left(v_{j}, Q_{\zeta_{1}, \zeta_{2}}^{\sharp}\right)\right| \leqslant \kappa\left(\left[v_{j}\right]_{K, \mathbb{R} \times \mathbb{R}}+\left\|v_{j}\right\|_{L^{2}(\mathbb{R})}\right) \leqslant \kappa\left(\varepsilon\left[v_{j}\right]_{K, \mathbb{R} \times \mathbb{R}}^{2}+\varepsilon\left\|v_{j}\right\|_{L^{2}(\mathbb{R})}^{2}+\frac{1}{\varepsilon}\right) .
$$

Hence, in view of 5.23 and choosing $\varepsilon$ conveniently small (possibly in dependence of $\mu$ ),

$$
\frac{3 \eta}{8}\left[v_{j}\right]_{H^{1}(\mathbb{R})}^{2} d x+\frac{\mu}{4}\left\|v_{j}\right\|_{L^{2}(\mathbb{R})}^{2}+\frac{1}{8}\left[v_{j}\right]_{K, \mathbb{R} \times \mathbb{R}}^{2} \leqslant \frac{\kappa}{\mu} .
$$

Accordingly, we obtain that, up to a subsequence, $v_{j}$ converges locally uniformly in $\mathbb{R}$ and weakly in $H^{1}(\mathbb{R})$ and in the Hilbert space induced by $[\cdot]_{K, \mathbb{R} \times \mathbb{R}}$ to a minimizer $v_{\eta, \mu}$. We then set $Q_{\eta, \mu}:=v_{\eta, \mu}+Q_{\zeta_{1}, \zeta_{2}}^{\sharp}$ and we obtain (5.9).

Also, the claims in (5.10), 5.11) and (5.13) follow by taking the limit in 5.25), as well as the claim in 5.12 follows by taking the limit in 5.20 . 
Moreover, the claim in (5.14) follows from 5.10 and the one in 5.15 is a consequence of (5.19). Finally, to prove 5.16 we observe that, in view of 2.2 , 2.3 and 5.24 ,

$$
E_{\mathbb{R}^{2}}\left(Q_{j}\right)=\left[v_{j}\right]_{K, \mathbb{R} \times \mathbb{R}}^{2}+2 \mathscr{B}_{\mathbb{R}, \mathbb{R}}\left(v_{j}, Q_{\zeta_{1}, \zeta_{2}}^{\sharp}\right) \geqslant \frac{1}{2}\left[v_{j}\right]_{K, \mathbb{R} \times \mathbb{R}}^{2}-\frac{1}{2}\left\|v_{j}\right\|_{L^{2}(\mathbb{R})}^{2}-\kappa .
$$

Passing to the limit and making use of (5.11) and 5.13) we obtain 5.16).

Now we define

$$
J_{*}:=\left(b_{1}, b_{2}\right)
$$

and

$$
L:=\left\{x \in\left(-\infty, b_{1}\right] \cup\left[b_{2},+\infty\right) \text { s.t. } \Psi(x)<Q_{\eta, \mu}(x)<\Phi(x)\right\} .
$$

Let also

$$
F:=J_{*} \cup L
$$

As usual, by taking inner variations, one sees that in the set $F$ the minimization problem is "free" and so it satisfies an Euler-Lagrange equation, as stated explicitly in the next result:

Lemma 5.2. Let $Q_{\eta, \mu}$ be as in Lemma5.1. For any $x \in F$, we have that

$$
-\eta \ddot{Q}_{\eta, \mu}(x)+\mu\left(Q_{\eta, \mu}(x)-Q_{\zeta_{1}, \zeta_{2}}^{\sharp}(x)\right)+\mathscr{L} Q_{\eta, \mu}(x)+a(x) W^{\prime}\left(Q_{\eta, \mu}(x)\right)=0 .
$$

Now we define the set

$\Sigma:=\left\{Q: \mathbb{R} \rightarrow \mathbb{R}\right.$ s.t. $Q-Q_{\zeta_{1}, \zeta_{2}}^{\sharp} \in H^{1}(\mathbb{R})$ and $\Psi(x) \leqslant Q(x) \leqslant \Phi(x)$ for all $\left.x \in \mathbb{R}\right\}$.

We notice that, differently from the definition of $\Gamma\left(b_{1}, b_{2}\right)$ given in 5.7), we require here that a function $Q$ belongs to $\Sigma$ if it satisfies $\Psi \leqslant Q \leqslant \Phi$ in the whole of $\mathbb{R}$, and not only in $\left(-\infty, b_{1}\right] \cup\left[b_{2},+\infty\right)$.

As a matter of fact, we prove that the minimizer $Q_{\eta, \mu} \in \Gamma\left(b_{1}, b_{2}\right)$, given by Lemma 5.1 is actually a minimizer of $I_{\eta, \mu}$ in $\Sigma$ :

Lemma 5.3. Let $Q_{\eta, \mu}$ be as in Lemma 5.1. Then, we have that $Q_{\eta, \mu} \in \Sigma$. In particular,

$$
\inf _{Q \in \Sigma} I_{\eta, \mu}(Q)=\inf _{Q \in \Gamma\left(b_{1}, b_{2}\right)} I_{\eta, \mu}(Q)=I_{\eta, \mu}\left(Q_{\eta, \mu}\right) .
$$

Proof. We first prove that $Q_{\eta, \mu}$ belongs to $\Sigma$. For this, it is enough to show that

$$
\Psi(x) \leqslant Q_{\eta, \mu}(x) \leqslant \Phi(x) \text { for any } x \in\left(b_{1}, b_{2}\right) .
$$

To check this, we observe that, by Lemma $5.2, Q_{\eta, \mu}$ is solution of

$$
-\eta \ddot{Q}_{\eta, \mu}(x)+\mu\left(Q_{\eta, \mu}(x)-Q_{\zeta_{1}, \zeta_{2}}^{\sharp}(x)\right)+\mathscr{L} Q_{\eta, \mu}(x)+a(x) W^{\prime}\left(Q_{\eta, \mu}(x)\right)=0
$$

for any $x \in\left(b_{1}, b_{2}\right)$. 
In addition, since $Q_{\eta, \mu} \in \Gamma\left(b_{1}, b_{2}\right)$, recalling 5.5 and 5.7 , we see that

$$
Q_{\eta, \mu}(x) \leqslant \Phi(x) \leqslant \phi(x) \quad \text { for any } x \in\left(-\infty, b_{1}\right] \cup\left[b_{2},+\infty\right) .
$$

We observe that

$$
Q_{\eta, \mu}(x) \leqslant \phi(x) \quad \text { for any } x \in\left(b_{1}, b_{2}\right) .
$$

To prove this, we define $w:=Q_{\eta, \mu}-\phi$ and we suppose, by contradiction, in light of (5.31), that $w$ has a positive maximum at some point $x_{\star} \in\left(b_{1}, b_{2}\right)$. This gives that

$$
0 \leqslant-\eta \ddot{w}\left(x_{\star}\right)+\mathscr{L} w\left(x_{\star}\right)=-\eta \ddot{Q}_{\eta, \mu}\left(x_{\star}\right)+\mathscr{L} Q_{\eta, \mu}\left(x_{\star}\right)+\eta \ddot{\phi}\left(x_{\star}\right)-\mathscr{L} \phi\left(x_{\star}\right) .
$$

Hence, recalling (5.1) and (5.27), and using also (5.15),

$$
\begin{aligned}
0 & \leqslant-\mu\left(Q_{\eta, \mu}\left(x_{\star}\right)-Q_{\zeta_{1}, \zeta_{2}}^{\sharp}\left(x_{\star}\right)\right)-a\left(x_{\star}\right) W^{\prime}\left(Q_{\eta, \mu}\left(x_{\star}\right)\right)-C_{0} \\
& \leqslant 2\left|\zeta_{1}\right|+2\left|\zeta_{2}\right|+\left\|a W^{\prime}\right\|_{L^{\infty}(\mathbb{R})}-C_{0} .
\end{aligned}
$$

This is in contradiction with (5.3) and hence it completes the proof of 5.32 .

Consequently, in view of (5.32), and making again use of (5.5),

$$
Q_{\eta, \mu}(x) \leqslant \phi(x) \leqslant \Phi(x) \text { for any } x \in\left(b_{1}, b_{2}\right),
$$

which proves the second inequality in 5.30 . Similarly, one can check that

$$
Q_{\eta, \mu}(x) \geqslant \Psi(x) \text { for any } x \in\left(b_{1}, b_{2}\right),
$$

which completes the proof of 5.30 .

Now, since $Q_{\eta, \mu} \in \Sigma \subset \Gamma\left(b_{1}, b_{2}\right)$, we have that

$$
\inf _{Q \in \Sigma} I_{\eta, \mu}(Q) \geqslant \inf _{Q \in \Gamma\left(b_{1}, b_{2}\right)} I_{\eta, \mu}(Q)=I_{\eta, \mu}\left(Q_{\eta, \mu}\right) \geqslant \inf _{Q \in \Sigma} I_{\eta, \mu}(Q),
$$

which proves $(5.29)$. The proof of Lemma 5.3 is thus complete.

\section{Lewy-Stampacchia estimates and continuity results for a double obstacle problem}

In this section, we prove that constrained minimizers of the perturbed problem are continuous, with uniform bounds. This estimate is based on a double obstacle problem approach. We follow a technique introduced by Lewy and Stampacchia in 27 and suitably modified in 39 to deal with nonlocal problems. In our situation, differently from the previous literature, we need to take into account the fact that the problem is constrained by two obstacles. Moreover, our problem is a superposition of a local and a nonlocal operators and we aim at estimates which are uniform 
with respect to the local contribution. The result that suits for our purposes is the following:

Proposition 6.1. Let $I$ be a bounded interval and $f \in L^{\infty}(I)$. Let $u \in \Sigma$, with $\Sigma$ defined as in (5.28), and assume that

$$
\begin{aligned}
\eta \int_{\mathbb{R}} & \dot{u}(x)(\dot{u}(x)-\dot{v}(x)) d x \\
& +\frac{1}{2} \iint_{\mathbb{R}^{2}}(u(x)-u(y))((u-v)(x)-(u-v)(y)) K(x-y) d x d y \\
& \leqslant \int_{\mathbb{R}} f(x)(u-v)(x) d x
\end{aligned}
$$

for every $v \in \Sigma$ with $v=u$ in $\mathbb{R} \backslash I$. Then,

$$
\begin{aligned}
\min \left\{\inf _{x \in I}-|\ddot{\Phi}(x)|+\mathscr{L} \Phi(x), \inf _{x \in I} f(x)\right\} & \leqslant-\eta \ddot{u}(x)+\mathscr{L} u(x) \\
& \leqslant \max \left\{\sup _{x \in I}|\ddot{\Psi}(x)|+\mathscr{L} \Psi(x), \sup _{x \in I} f(x)\right\}
\end{aligned}
$$

in the sense of distributions.

Proof. Let

$$
M^{*}:=\max \left\{\sup _{x \in I}|\ddot{\Psi}(x)|+\mathscr{L} \Psi(x), \sup _{x \in I} f(x)\right\}
$$

and

$$
I^{*}(v):=\frac{\eta}{2} \int_{I}|\dot{v}(x)|^{2} d x+\frac{1}{4} \iint_{Q_{I}}|v(x)-v(y)|^{2} K(x-y) d x d y-M^{*} \int_{I} v(x) d x,
$$

where $Q_{I}:=(I \times I) \cup(I \times(\mathbb{R} \backslash I)) \cup((\mathbb{R} \backslash I) \times I)$. We take $z^{*}$ to be a minimizer of $I^{*}$ in the class of functions $v: \mathbb{R} \rightarrow \mathbb{R}$ with $v(x) \leqslant u(x)$ for any $x \in \mathbb{R}$ and $v(x)=u(x)$ for any $x \in \mathbb{R} \backslash I$. The existence of such minimizer follows by compactness, along the lines given in the proof of Lemma 5.1. In particular,

$$
z^{*}(x) \leqslant u(x) \text { for any } x \in \mathbb{R} \text { and } z^{*}(x)=u(x) \text { for any } x \in \mathbb{R} \backslash I \text {. }
$$

Moreover, for any $\varepsilon \in[0,1]$ and any $w: \mathbb{R} \rightarrow \mathbb{R}$ with $w(x) \leqslant u(x)$ for any $x \in \mathbb{R}$ and $w(x)=u(x)$ for any $x \in \mathbb{R} \backslash I$, we have that $z_{\varepsilon}(x):=\varepsilon w(x)+(1-\varepsilon) z^{*}(x)$ is an 
admissible competitor for $z^{*}$ and consequently $I^{*}\left(z_{\varepsilon}\right) \geqslant I^{*}\left(z^{*}\right)$, which gives that

$$
\begin{aligned}
0 \leqslant\left.\frac{d}{d \varepsilon} I^{*}\left(z_{\varepsilon}\right)\right|_{\varepsilon=0} & \\
=\eta \int_{I} \dot{z}^{*}(x) & \left(\dot{w}(x)-\dot{z}^{*}(x)\right) d x \\
& +\frac{1}{2} \iint_{Q_{I}}\left(z^{*}(x)-z^{*}(y)\right)\left(\left(w-z^{*}\right)(x)-\left(w-z^{*}\right)(y)\right) K(x-y) d x d y \\
& -M^{*} \int_{I}\left(w-z^{*}\right)(x) d x .
\end{aligned}
$$

We claim that

$$
z^{*} \in \Sigma
$$

To check this, we first use 6.4 to observe that

$$
z^{*}(x) \leqslant u(x) \leqslant \Phi(x)
$$

Then, we take

$$
w^{*}(x):=\max \left\{z^{*}(x), \Psi(x)\right\}=z^{*}(x)+\left(\Psi(x)-z^{*}(x)\right)_{+} .
$$

By 6.4, we know that $w^{*}(x) \leqslant u(x)$ for any $x \in \mathbb{R}$. Also, if $x \in \mathbb{R} \backslash I$, we have that $w^{*}(x)=\max \{u(x), \Psi(x)\}=u(x)$. Therefore, we can make use of 6.5 with $w:=w^{*}$, and so we find that

$$
\begin{aligned}
0 & \leqslant \eta \int_{I \cap\left\{\Psi>z^{*}\right\}} \dot{z}^{*}(x)\left(\dot{\Psi}(x)-\dot{z}^{*}(x)\right) d x \\
& +\frac{1}{2} \iint_{Q_{I}}\left(z^{*}(x)-z^{*}(y)\right)\left(\left(\Psi(x)-z^{*}(x)\right)_{+}-\left(\Psi(y)-z^{*}(y)\right)_{+}\right) K(x-y) d x d y \\
& -M^{*} \int_{I}\left(\Psi(x)-z^{*}(x)\right)_{+} d x .
\end{aligned}
$$

Furthermore, on $\partial I$ we have that $z^{*}=u \geqslant \Psi$, hence, from (6.3) and integrating by 
parts, we see that

$$
\begin{aligned}
& \eta \int_{I \cap\left\{\Psi>z^{*}\right\}} \dot{\Psi}(x)\left(\dot{\Psi}(x)-\dot{z}^{*}(x)\right) d x \\
& +\frac{1}{2} \iint_{Q_{I}}(\Psi(x)-\Psi(y))\left(\left(\Psi(x)-z^{*}(x)\right)_{+}-\left(\Psi(y)-z^{*}(y)\right)_{+}\right) K(x-y) d x d y \\
& -M^{*} \int_{I}\left(\Psi(x)-z^{*}(x)\right)_{+} d x \\
= & -\eta \int_{\mathbb{R}} \ddot{\Psi}(x)\left(\Psi(x)-z^{*}(x)\right)_{+} d x \\
& +\iint_{\mathbb{R}^{2}}(\Psi(x)-\Psi(y))\left(\Psi(x)-z^{*}(x)\right)_{+} K(x-y) d x d y \\
& -M^{*} \int_{\mathbb{R}}\left(\Psi(x)-z^{*}(x)\right)_{+} d x \\
= & \int_{\mathbb{R}}\left(-\eta \ddot{\Psi}(x)+\mathscr{L} \Psi(x)-M^{*}\right)\left(\Psi(x)-z^{*}(x)\right)_{+} d x \\
\leqslant & 0
\end{aligned}
$$

Thus, subtracting (6.8) to 6.9), we conclude that

$$
\begin{aligned}
0 \geqslant & \eta \int_{I}\left(\dot{\Psi}(x)-\dot{z}^{*}(x)\right)\left(\dot{\Psi}(x)-\dot{z}^{*}(x)\right)_{+} d x \\
& \quad+\frac{1}{2} \iint_{Q_{I}}\left(\left(\Psi(x)-z^{*}(x)\right)-\left(\Psi(y)-z^{*}(y)\right)\right) \\
& \times\left(\left(\Psi(x)-z^{*}(x)\right)_{+}-\left(\Psi(y)-z^{*}(y)\right)_{+}\right) K(x-y) d x d y .
\end{aligned}
$$

The last term in 6.10 is nonnegative (see e.g. page 1115 in 39$]$ ), therefore we get that

$$
0 \geqslant \int_{I}\left(\dot{\Psi}(x)-\dot{z}^{*}(x)\right)_{+}^{2} d x
$$

This says that $\Psi(x) \leqslant z^{*}(x)$ for any $x \in I$ (and so for any $x \in \mathbb{R}$, due to 6.4 ). This and 6.7) imply 6.6), as desired.

Then, from (6.6) we deduce that both the minimum and the maximum between $u$ and $z^{*}$ belong to $\Sigma$, that is

$$
\begin{gathered}
v^{\sharp}(x):=\min \left\{u(x), z^{*}(x)\right\}=u(x)-\left(u(x)-z^{*}(x)\right)_{+} \in \Sigma \\
\text { and } \quad w^{\sharp}(x):=\max \left\{u(x), z^{*}(x)\right\}=z^{*}(x)+\left(u(x)-z^{*}(x)\right)_{+} \in \Sigma .
\end{gathered}
$$


In particular, we can take $v:=v^{\sharp}$ in (6.1) and $w:=w^{\sharp}$ in 6.5. This gives that

$$
\begin{aligned}
\eta \int_{\mathbb{R}} & \dot{u}(x)\left(\dot{u}(x)-\dot{z}^{*}(x)\right)_{+} d x \\
& +\frac{1}{2} \iint_{\mathbb{R}^{2}}(u(x)-u(y))\left(\left(u(x)-z^{*}(x)\right)_{+}-\left(u(y)-z^{*}(y)\right)_{+}\right) K(x-y) d x d y \\
& \leqslant \int_{\mathbb{R}} f(x)\left(u(x)-z^{*}(x)\right)_{+} d x
\end{aligned}
$$

and

$$
\begin{aligned}
& M^{*} \int_{I}\left(u(x)-z^{*}(x)\right)_{+} d x \leqslant \eta \int_{I} \dot{z}^{*}(x)\left(\dot{u}(x)-\dot{z}^{*}(x)\right)_{+} d x \\
& +\frac{1}{2} \iint_{Q_{I}}\left(z^{*}(x)-z^{*}(y)\right)\left(\left(u(x)-z^{*}(x)\right)_{+}-\left(u(y)-z^{*}(y)\right)_{+}\right) K(x-y) d x d y .
\end{aligned}
$$

Hence, subtracting (6.12) to 6.11) and recalling 6.3), we obtain

$$
\begin{aligned}
0 \geqslant & \eta \int_{I}\left(\dot{u}(x)-\dot{z}^{*}(x)\right)\left(\dot{u}(x)-\dot{z}^{*}(x)\right)_{+} d x \\
& +\frac{1}{2} \iint_{Q_{I}}\left(\left(u(x)-z^{*}(x)\right)-\left(u(y)-z^{*}(y)\right)\right) \\
\times & \left(\left(u(x)-z^{*}(x)\right)_{+}-\left(u(y)-z^{*}(y)\right)_{+}\right) K(x-y) d x d y .
\end{aligned}
$$

As above, this implies that $u \leqslant z^{*}$. Combining this with $(6.4)$, we obtain that $z^{*}$ coincides with $u$. As a consequence, taking any function $v \geqslant 0$, supported in $I$, and defining $w:=u-v$ in 6.5,

$\eta \int_{I} \dot{u}(x) \dot{v}(x) d x+\frac{1}{2} \iint_{Q_{I}}(u(x)-u(y))(v(x)-v(y)) K(x-y) d x d y \leqslant M^{*} \int_{I} v(x) d x$.

Integrating by parts the latter inequality, we obtain that

$$
\int_{\mathbb{R}}(-\eta \ddot{u}(x)+\mathscr{L} u(x)) v(x) d x \leqslant M^{*} \int_{\mathbb{R}} v(x) d x .
$$

By duality, we thus obtain that

$$
-\eta \ddot{u}(x)+\mathscr{L} u(x) \leqslant M^{*},
$$

in the sense of distributions, which is one of the inequalities in 6.2. The other inequality in 6.2 follows by similar arguments.

Using Lemma 3.1. Proposition 6.1 and a convolution method (see e.g. formula (3.2) in [40]), we obtain a useful uniform continuity result for a perturbed problem. The statement that we need for our purposes is the following:

Corollary 6.1. Let $Q_{\eta, \mu}$ be as in Lemma 5.1 and $\alpha \in(0,2 s)$. Then $Q_{\eta, \mu} \in C^{0, \alpha}(\mathbb{R})$ and

$$
\left\|Q_{\eta, \mu}\right\|_{C^{0, \alpha}(\mathbb{R})} \leqslant \kappa
$$


for some $\kappa>0$, which possibly depends on $Q_{\zeta_{1}, \zeta_{2}}^{\sharp}$ and on structural constants.

Proof. We take $v_{\eta, \mu}:=Q_{\eta, \mu}-Q_{\zeta_{1}, \zeta_{2}}^{\sharp}$, as in Lemma 5.1. By Lemma 5.3, we know that $Q_{\eta, \mu} \in \Sigma$. We fix an interval $I \subset \mathbb{R}$ and take any $\xi \in \Sigma$. For any $\varepsilon \in(0,1)$, let $\xi_{\varepsilon}:=\varepsilon \xi+(1-\varepsilon) Q_{\eta, \mu}=Q_{\eta, \mu}+\varepsilon\left(\xi-Q_{\eta, \mu}\right)$. Then $\xi_{\varepsilon} \in \Sigma$ and therefore, by (5.29), we know that

$$
\begin{aligned}
0 \leqslant & I_{\eta, \mu}\left(\xi_{\varepsilon}\right)-I_{\eta, \mu}\left(Q_{\eta, \mu}\right) \\
= & \frac{\eta}{2} \int_{I}\left(\left|\dot{\xi}_{\varepsilon}(x)\right|^{2}-\left|\dot{Q}_{\eta, \mu}(x)\right|^{2}\right) d x \\
& +\frac{\mu}{2} \int_{I}\left(\left|\xi_{\varepsilon}(x)-Q_{\zeta_{1}, \zeta_{2}}^{\sharp}(x)\right|^{2}-\left|Q_{\eta, \mu}(x)-Q_{\zeta_{1}, \zeta_{2}}^{\sharp}(x)\right|^{2}\right) d x \\
& +\int_{I} a(x)\left(W\left(\xi_{\varepsilon}(x)\right)-W\left(Q_{\eta, \mu}(x)\right)\right) d x \\
& +\frac{1}{4} \iint_{\mathbb{R} \times \mathbb{R}}\left(\left|\xi_{\varepsilon}(x)-\xi_{\varepsilon}(y)\right|^{2}-\left|Q_{\eta, \mu}(x)-Q_{\eta, \mu}(y)\right|^{2}\right) K(x-y) d x d y \\
= & \varepsilon \eta \int_{I} \dot{Q}_{\eta, \mu}(x) \cdot\left(\dot{\xi}(x)-\dot{Q}_{\eta, \mu}(x)\right) d x \\
& +\varepsilon \mu \int_{I}\left(Q_{\eta, \mu}(x)-Q_{\zeta_{1}, \zeta_{2}}^{\sharp}(x)\right) \cdot\left(\xi(x)-Q_{\eta, \mu}(x)\right) d x \\
& +\varepsilon \int_{I} a(x) W^{\prime}\left(Q_{\eta, \mu}(x)\right)\left(\xi(x)-Q_{\eta, \mu}(x)\right) d x \\
& +\frac{\varepsilon}{2} \iint_{\mathbb{R} \times \mathbb{R}}\left(\left(Q_{\eta, \mu}(x)-Q_{\eta, \mu}(y)\right)\left(\left(\xi-Q_{\eta, \mu}\right)(x)-\left(\xi-Q_{\eta, \mu}\right)(y)\right)\right) K(x-y) d x d y \\
& +o(\varepsilon) .
\end{aligned}
$$

Thus, dividing this inequality by $\varepsilon$ and sending $\varepsilon \searrow 0$, we conclude that $Q_{\eta, \mu}$ satisfies (6.1) with

$$
f:=-a W^{\prime}\left(Q_{\eta, \mu}\right)-\mu\left(Q_{\eta, \mu}-Q_{\zeta_{1}, \zeta_{2}}^{\sharp}\right) .
$$

Accordingly, by formula 6.2 in Proposition 6.1. we know that

$$
- \text { const } \leqslant-\eta \ddot{Q}_{\eta, \mu}+\mathscr{L} Q_{\eta, \mu} \leqslant \text { const }
$$

and therefore

$$
-\kappa \leqslant-\eta \ddot{v}_{\eta, \mu}+\mathscr{L} v_{\eta, \mu} \leqslant \kappa
$$

in the sense of distributions, for some $\kappa>0$, which possibly depends on $Q_{\zeta_{1}, \zeta_{2}}^{\sharp}$.

Now we take an even function $\vartheta \in C_{0}^{\infty}([-1,1])$ and for any $\varepsilon \in(0,1)$ we set $\vartheta_{\varepsilon}(x):=\varepsilon^{-1} \vartheta(x / \varepsilon)$. We consider the mollification $v_{\eta, \mu, \varepsilon}:=v_{\eta, \mu} * \vartheta_{\varepsilon}$. Notice that, as $\varepsilon \searrow 0$, we have that

$$
v_{\eta, \mu, \varepsilon} \text { converges locally uniformly to } v_{\eta, \mu},
$$


thanks to (5.14). Moreover, we observe that, for any $\varphi \in C_{0}^{\infty}(\mathbb{R})$,

$$
\begin{aligned}
& \left|\left(v_{\eta, \mu}(x)-v_{\eta, \mu}(y)\right)(\varphi(x)-\varphi(y)) \vartheta_{\varepsilon}(z) K(x-y)\right| \\
& \quad \leqslant\left(\left|v_{\eta, \mu}(x)-v_{\eta, \mu}(y)\right|^{2} K(x-y)+|\varphi(x)-\varphi(y)|^{2} K(x-y)\right) \chi_{[-1,1]}(z),
\end{aligned}
$$

which, as a function of $(x, y, z) \in \mathbb{R} \times \mathbb{R} \times \mathbb{R}$, belongs to $L^{1}(\mathbb{R} \times \mathbb{R} \times \mathbb{R})$, thanks to (5.11). This implies that we can exploit the Dominated Convergence Theorem and obtain that

$$
\begin{aligned}
& \iint_{\mathbb{R} \times \mathbb{R}}\left(v_{\eta, \mu, \varepsilon}(x)-v_{\eta, \mu, \varepsilon}(y)\right)(\varphi(x)-\varphi(y)) K(x-y) d x d y \\
= & \iint_{\mathbb{R} \times \mathbb{R}}\left[\int_{\mathbb{R}}\left(v_{\eta, \mu}(x-z)-v_{\eta, \mu}(y-z)\right) \vartheta_{\varepsilon}(z)(\varphi(x)-\varphi(y)) K(x-y) d z\right] d x d y \\
= & \int_{\mathbb{R}}\left[\iint_{\mathbb{R} \times \mathbb{R}}\left(v_{\eta, \mu}(x-z)-v_{\eta, \mu}(y-z)\right) \vartheta_{\varepsilon}(z)(\varphi(x)-\varphi(y)) K(x-y) d x d y\right] d z \\
= & \int_{\mathbb{R}}\left[\iint_{\mathbb{R} \times \mathbb{R}}\left(v_{\eta, \mu}(x)-v_{\eta, \mu}(y)\right) \vartheta_{\varepsilon}(z)(\varphi(x+z)-\varphi(y+z)) K(x-y) d x d y\right] d z \\
= & \iint_{\mathbb{R} \times \mathbb{R}}\left[\int_{\mathbb{R}}\left(v_{\eta, \mu}(x)-v_{\eta, \mu}(y)\right) \vartheta_{\varepsilon}(z)(\varphi(x+z)-\varphi(y+z)) K(x-y) d z\right] d x d y \\
= & \iint_{\mathbb{R} \times \mathbb{R}}\left[\int_{\mathbb{R}}\left(v_{\eta, \mu}(x)-v_{\eta, \mu}(y)\right) \vartheta_{\varepsilon}(z)(\varphi(x-z)-\varphi(y-z)) K(x-y) d z\right] d x d y \\
= & \iint_{\mathbb{R} \times \mathbb{R}}\left(v_{\eta, \mu}(x)-v_{\eta, \mu}(y)\right)\left(\varphi_{\varepsilon}(x)-\varphi_{\varepsilon}(y)\right) K(x-y) d x d y,
\end{aligned}
$$

where $\varphi_{\varepsilon}:=\varphi * \vartheta_{\varepsilon}$. Similarly, by (5.10), we see that

$$
\int_{\mathbb{R}} \dot{v}_{\eta, \mu, \varepsilon}(x) \dot{\varphi}(x) d x=\int_{\mathbb{R}} \dot{v}_{\eta, \mu}(x) \dot{\varphi}_{\varepsilon}(x) d x .
$$

From 6.14, 6.16) and (6.17) we infer that, for any $\varphi \in C_{0}^{\infty}(\mathbb{R},[0,1])$,

$$
\begin{aligned}
& \left|\eta \int_{\mathbb{R}} \dot{v}_{\eta, \mu, \varepsilon}(x) \dot{\varphi}(x) d x+\frac{1}{2} \iint_{\mathbb{R} \times \mathbb{R}}\left(v_{\eta, \mu, \varepsilon}(x)-v_{\eta, \mu, \varepsilon}(y)\right)(\varphi(x)-\varphi(y)) K(x-y) d x d y\right| \\
& \quad=\left|\eta \int_{\mathbb{R}} \dot{v}_{\eta, \mu}(x) \dot{\varphi}_{\varepsilon}(x) d x+\frac{1}{2} \iint_{\mathbb{R} \times \mathbb{R}}\left(v_{\eta, \mu}(x)-v_{\eta, \mu}(y)\right)\left(\varphi_{\varepsilon}(x)-\varphi_{\varepsilon}(y)\right) K(x-y) d x d y\right| \\
& \quad \leqslant \kappa\left|\int_{\mathbb{R}} \varphi_{\varepsilon}(x) d x\right| \leqslant \kappa \int_{\mathbb{R}} \varphi(x) d x .
\end{aligned}
$$

That is,

$$
-\kappa \leqslant-\eta \ddot{v}_{\eta, \mu, \varepsilon}+\mathscr{L} v_{\eta, \mu, \varepsilon} \leqslant \kappa
$$

in the sense of distributions, and also in the classical and viscosity senses, since $v_{\eta, \mu, \varepsilon}$ is smooth. Therefore, we are in the position of applying Lemma 3.1 to $v_{\eta, \mu, \varepsilon}$ and conclude that

$$
\begin{aligned}
{\left[v_{\eta, \mu, \varepsilon}\right]_{C^{0, \alpha}(\mathbb{R})} } & \leqslant \kappa\left(1+\left\|v_{\eta, \mu, \varepsilon}\right\|_{L^{\infty}(\mathbb{R})}\right)^{\frac{\alpha}{2 s}}\left\|v_{\eta, \mu, \varepsilon}\right\|_{L^{\infty}(\mathbb{R})}^{1-\frac{\alpha}{2 s}} \\
& \leqslant \kappa\left(1+\left\|v_{\eta, \mu}\right\|_{L^{\infty}(\mathbb{R})}\right)^{\frac{\alpha}{2 s}}\left\|v_{\eta, \mu}\right\|_{L^{\infty}(\mathbb{R})}^{1-\frac{\alpha}{2 s}},
\end{aligned}
$$


for any $\alpha \in(0,2 s)$ (up to freely renaming $\kappa$ ). As a consequence of this and 5.12 , we obtain that $\left[v_{\eta, \mu, \varepsilon}\right]_{C^{0, \alpha}(\mathbb{R})} \leqslant \kappa$. This and 6.15 imply that $\left[v_{\eta, \mu}\right]_{C^{0, \alpha}(\mathbb{R})} \leqslant \kappa$. Using this and 5.12, we obtain that $\left\|v_{\eta, \mu}\right\|_{C^{0, \alpha}(\mathbb{R})} \leqslant \kappa$, which in turn implies [6.13), as desired.

\section{Clean intervals and clean points}

Here we deal with the notions of clean intervals and clean points, which have been introduced in Section 6 of 21$]$ to perform glueing techniques in the nonlocal setting.

Definition 7.1. Given $\rho>0$ and $Q: \mathbb{R} \rightarrow \mathbb{R}$, we say that an interval $J \subseteq \mathbb{R}$ is a "clean interval" for $(\rho, Q)$ if $|J| \geqslant|\log \rho|$ and there exists $\zeta \in \mathscr{Z}$ such that

$$
\sup _{x \in J}|Q(x)-\zeta| \leqslant \rho .
$$

Definition 7.2. If $J$ is a bounded clean interval for $(\rho, Q)$, the center of $J$ is called a "clean point" for $(\rho, Q)$.

Here we show that any sufficiently large interval contains a clean interval.

Lemma 7.1. Let $Q_{\eta, \mu}$ be as in Lemma 5.1. Then, there exist $\rho_{0} \in(0,1)$ and $\kappa_{1}>0$ depending on $Q_{\zeta_{1}, \zeta_{2}}^{\sharp}$ and on the structural constants such that, if $\rho \in\left(0, \rho_{0}\right)$ and $J \subseteq \mathbb{R}$ is an interval such that

$$
|J| \geqslant \frac{\kappa_{1}\left[Q_{\eta, \mu}\right]_{C^{0, \alpha}(J)}^{\frac{1}{\alpha}}}{\mu^{2} \rho^{2+\frac{1}{\alpha}}}|\log \rho|,
$$

for $\alpha \in(0,2 s)$, then there exists a clean interval for $\left(\rho, Q_{\eta, \mu}\right)$ that is contained in $J$.

Proof. By Corollary 6.1, we know that $Q_{\eta, \mu} \in C^{0, \alpha}(J)$ for any $\alpha \in(0,2 s)$. Without loss of generality we can assume that $\left[Q_{\eta, \mu}\right]_{C^{0, \alpha}(J)} \geqslant 1$. Assume, by contradiction, that

$$
J \text { does not contain any clean subinterval. }
$$

By (7.1), the interval $J$ contains $N$ disjoint subintervals, say $J_{1}, \ldots, J_{N}$, each of length $|\log \rho|$, with

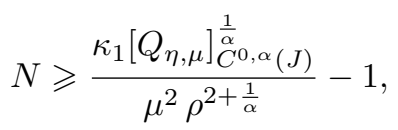

and, by $(7.2)$, none of the subintervals $J_{i}$ is clean. Hence, for any $i \in\{1, \ldots, N\}$, there exists $p_{i} \in J_{i}$ such that $Q\left(p_{i}\right)$ stays at distance larger than $\rho$ from $\mathscr{Z}$. Also, letting

$$
\ell_{\rho}:=\left(\frac{\rho}{2\left[Q_{\eta, \mu}\right]_{C^{0, \alpha}(J)}}\right)^{\frac{1}{\alpha}},
$$


we have that, for any $x \in J_{i}^{\prime}:=\left[p_{i}-\ell_{\rho}, p_{i}+\ell_{\rho}\right]$,

$$
\left|Q_{\eta, \mu}(x)-Q_{\eta, \mu}\left(p_{i}\right)\right| \leqslant\left[Q_{\eta, \mu}\right]_{C^{0, \alpha}(J)}\left|x-p_{i}\right|^{\alpha} \leqslant\left[Q_{\eta, \mu}\right]_{C^{0, \alpha}(J)} \ell_{\rho}^{\alpha}=\frac{\rho}{2} .
$$

Accordingly, $Q_{\eta, \mu}(x)$ stays at distance larger than $\frac{\rho}{2}$ from $\mathscr{Z}$ for any $x \in J_{i}^{\prime}$ and then, by (1.8),

$$
W\left(Q_{\eta, \mu}(x)\right) \geqslant \frac{c_{0} \rho^{2}}{4} .
$$

Moreover, for $\rho$ sufficiently small, at least half of the interval $J_{i}^{\prime}$ lies in $J_{i}$, hence

$$
\int_{J_{i} \cap J_{i}^{\prime}} W\left(Q_{\eta, \mu}(x)\right) d x \geqslant \frac{c_{0} \rho^{2} \ell_{\rho}}{4}=\frac{\kappa \rho^{2+\frac{1}{\alpha}}}{\left[Q_{\eta, \mu}\right]_{C^{0, \alpha}(J)}^{\frac{1}{\alpha}}} .
$$

Summing up over $i=1, \ldots, N$, using that the intervals $J_{i}$ are disjoint and recalling $(1.10),(5.9)$ and (5.16), we find that

$$
\begin{aligned}
I_{\eta, \mu} Q_{\zeta_{1}, \zeta_{2}}^{\sharp} & \geqslant I_{\eta, \mu}\left(Q_{\eta, \mu}\right) \\
& \geqslant-\frac{\kappa}{\mu^{2}}+\sum_{i=1}^{N} \int_{J_{i} \cap J_{i}^{\prime}} a(x) W\left(Q_{\eta, \mu}(x)\right) d x \\
& \geqslant-\frac{\kappa}{\mu^{2}}+\frac{N \underline{a} \kappa \rho^{2+\frac{1}{\alpha}}}{\left[Q_{\eta, \mu}\right]_{C^{0, \alpha}(J)}^{\frac{1}{\alpha}}},
\end{aligned}
$$

which gives

$$
N \leqslant \frac{\kappa\left[Q_{\eta, \mu}\right]_{C^{0, \alpha}(J)}^{\frac{1}{\alpha}}}{\mu^{2} \rho^{2+\frac{1}{\alpha}}} .
$$

This is a contradiction with 7.3 for $\kappa_{1}>\kappa+1$ and so it proves the desired result.

Lemma 7.2. Let $Q_{\eta, \mu}$ be as in Lemma 5.1. Let $T>1$ and $J:=\left(x_{0}-4 T, x_{0}+4 T\right)$ be a clean interval for $\left(\rho, Q_{\eta, \mu}\right)$. Then, for any $\alpha \in(0,2 s)$,

$$
\left[Q_{\eta, \mu}\right]_{C^{0, \alpha}\left(x_{0}-T, x_{0}+T\right)} \leqslant C\left(\frac{\rho^{1-\frac{\alpha}{2 s}}}{|\log \rho|^{\alpha}}+\rho+\mu^{\frac{\alpha}{2 s}} \rho^{1-\frac{\alpha}{2 s}}\right),
$$

for some $C>0$, independent of $\eta$ and $\mu$.

Proof. Let $\zeta \in \mathscr{Z}$ be such that $\sup _{x \in J}\left|Q_{\eta, \mu}(x)-\zeta\right| \leqslant \rho$. Then, according to Definition 7.1, we have that

$$
T \geqslant \frac{|\log \rho|}{8},
$$

and $J \subset F$, where $F$ is defined as in 5.26). Therefore, by Lemma 5.2, $Q_{\eta, \mu}$ is solution of

$$
-\eta, \ddot{Q}_{\eta, \mu}+\mu\left(Q_{\eta, \mu}-Q_{\zeta_{1}, \zeta_{2}}^{\sharp}\right)+\mathscr{L} Q_{\eta, \mu}+a W^{\prime}\left(Q_{\eta, \mu}\right)=0 \quad \text { in } J .
$$


Then by Lemma 3.2, (5.15) and (7.4), for $\alpha<2 s$, we have that

$$
\begin{aligned}
{\left[Q_{\eta, \mu}\right]_{C^{0, \alpha}\left(x_{0}-T, x_{0}+T\right)} } & \leqslant C T^{-\alpha}\left(1+T^{2 s} \rho+T^{2 s} \mu\right)^{\frac{\alpha}{2 s}} \rho^{1-\frac{\alpha}{2 s}} \\
& \leqslant C T^{-\alpha}\left(1+T^{\alpha} \rho^{\frac{\alpha}{2 s}}+T^{\alpha} \mu^{\frac{\alpha}{2 s}}\right) \rho^{1-\frac{\alpha}{2 s}} \\
& \leqslant C\left(T^{-\alpha} \rho^{1-\frac{\alpha}{2 s}}+\rho+\mu^{\frac{\alpha}{2 s}} \rho^{1-\frac{\alpha}{2 s}}\right) \\
& \leqslant C\left(\frac{\rho^{1-\frac{\alpha}{2 s}}}{\mid \log \rho^{\alpha}}+\rho+\mu^{\frac{\alpha}{2 s}} \rho^{1-\frac{\alpha}{2 s}}\right)
\end{aligned}
$$

by possibly renaming $C$. This proves the desired estimate of Lemma 7.2 .

Remark 7.1. Given $x_{0} \in \mathbb{R}$ and $\beta \in(1,+\infty)$, let $P: \mathbb{R} \rightarrow \mathbb{R}$ be a function such that

$$
v:=P-Q_{\zeta_{1}, \zeta_{2}}^{\sharp} \in H^{1}(\mathbb{R})
$$

and $P$ is Hölder continuous in $\left(x_{0}-\beta, x_{0}+\beta\right)$, with

$$
[P]_{C^{0, \alpha}\left(x_{0}-\beta, x_{0}+\beta\right)} \leqslant \delta,
$$

for some $\delta>0$. Given $T_{1}, T_{2}$ such that $-\infty \leqslant T_{1} \leqslant x_{0}-\beta<x_{0}+\beta \leqslant T_{2} \leqslant+\infty$, let us denote

$$
I_{-}:=\left(T_{1}, x_{0}\right), \quad I_{+}:=\left(x_{0}, T_{2}\right)
$$

and

$J_{-}:=\left(T_{1}, x_{0}-\beta\right), \quad D_{-}:=\left(x_{0}-\beta, x_{0}\right), \quad D_{+}:=\left(x_{0}, x_{0}+\beta\right), \quad J_{+}:=\left(x_{0}+\beta, T_{2}\right)$.

We want to estimate $E_{\left(T_{1}, T_{2}\right)^{2}}(P)$ in terms of $E_{I_{-}^{2}}(P)$ and $E_{I_{+}^{2}}(P)$. We have that

$$
E_{\left(T_{1}, T_{2}\right)^{2}}(P)=E_{I_{-}^{2}}(P)+E_{I_{+}^{2}}(P)+2 E_{I_{-} \times I_{+}}(P)
$$

and

$$
E_{I_{-} \times I_{+}}(P)=E_{J_{-} \times I_{+}}(P)+E_{D_{-} \times D_{+}}(P)+E_{D_{-} \times J_{+}}(P) .
$$

By (7.6) and (1.4,

$$
\begin{aligned}
0 \leqslant E_{D_{-} \times D_{+}}(P)+\left[Q_{\zeta_{1}, \zeta_{2}}^{\sharp}\right]_{K, D_{-} \times D_{+}}^{2} & =\int_{x_{0}-\beta}^{x_{0}} \int_{x_{0}}^{x_{0}+\beta}|P(x)-P(y)|^{2} K(x-y) d x d y \\
& \leqslant \Theta_{0} \delta^{2} \int_{x_{0}-\beta}^{x_{0}} \int_{x_{0}}^{x_{0}+\beta}|x-y|^{2 \alpha-1-2 s} d x d y \\
& \leqslant \kappa \delta^{2} \beta^{2 \alpha+1-2 s} .
\end{aligned}
$$

Moreover, recalling (7.5), we have that

$$
E_{J_{-} \times I_{+}}(P)=[v]_{K, J_{-} \times I_{+}}^{2}+2 \mathscr{B}_{J_{-} \times I_{+}}\left(v, Q_{\zeta_{1}, \zeta_{2}}^{\sharp}\right) .
$$


Now, by (1.4),

$$
\begin{aligned}
& \left|\mathscr{B}_{J_{-} \times I_{+}}\left(v, Q_{\zeta_{1}, \zeta_{2}}^{\sharp}\right)\right|=\left|\int_{T_{1}}^{x_{0}-\beta} \int_{x_{0}}^{T_{2}}(v(x)-v(y))\left(Q_{\zeta_{1}, \zeta_{2}}^{\sharp}(x)-Q_{\zeta_{1}, \zeta_{2}}^{\sharp}(y)\right) K(x-y) d x d y\right| \\
& \leqslant 2 \Theta_{0}\left\|Q_{\zeta_{1}, \zeta_{2}}^{\sharp}\right\|_{L^{\infty}(\mathbb{R})} \int_{-\infty}^{x_{0}-\beta} \int_{x_{0}}^{+\infty}(|v(x)|+|v(y)|)|x-y|^{-1-2 s} d x d y \\
& \quad=\frac{\Theta_{0}\left\|Q_{\zeta_{1}, \zeta_{2}}^{\sharp}\right\|_{L^{\infty}(\mathbb{R})}}{s}\left[\int_{-\infty}^{x_{0}-\beta}|v(x)|\left(x_{0}-x\right)^{-2 s} d x+\int_{x_{0}}^{+\infty}|v(y)|\left(y-x_{0}+\beta\right)^{-2 s} d y\right] .
\end{aligned}
$$

In addition, using the Cauchy-Schwarz inequality and (1.5), we see that

$$
\begin{aligned}
& \left.\int_{-\infty}^{x_{0}-\beta}|v(x)|\left(x_{0}-x\right)^{-2 s} d x \leqslant\left(\int_{-\infty}^{x_{0}-\beta}|v(x)|^{2} d x\right)\right)^{\frac{1}{2}}\left(\int_{-\infty}^{x_{0}-\beta}\left(x_{0}-x\right)^{-4 s} d x\right)^{\frac{1}{2}} \\
& \leqslant \kappa\|v\|_{L^{2}(\mathbb{R})} \beta^{-\frac{4 s-1}{2}} .
\end{aligned}
$$

Similarly,

$$
\int_{x_{0}}^{+\infty}|v(y)|\left(y-x_{0}+\beta\right)^{-2 s} d y \leqslant \kappa\|v\|_{L^{2}(\mathbb{R})} \beta^{-\frac{4 s-1}{2}} .
$$

Plugging these pieces of information into 7.10 , we have that

$$
\left|E_{J_{-} \times I_{+}}(P)\right| \leqslant[v]_{K,\left(-\infty, x_{0}-\beta\right) \times\left(x_{0},+\infty\right)}^{2}+\kappa\|v\|_{L^{2}(\mathbb{R})} \beta^{-\frac{4 s-1}{2}}
$$

Similar computations give

$$
\left|E_{D_{-} \times J_{+}}(P)\right| \leqslant[v]_{K,\left(x_{0}-\beta, x_{0}\right) \times\left(x_{0}+\beta,+\infty\right)}^{2}+\kappa\|v\|_{L^{2}(\mathbb{R})} \beta^{-\frac{4 s-1}{2}}
$$

Hence, from (7.8), 77.9), 7.11) and 7.12), we conclude that

$$
\begin{aligned}
& \quad\left|E_{I_{-} \times I_{+}}(P)+\left[Q_{\zeta_{1}, \zeta_{2}}^{\sharp}\right]_{K,\left(x_{0}-\beta, x_{0}\right) \times\left(x_{0}, x_{0}+\beta\right)}^{2}\right| \\
& \leqslant \kappa \delta^{2} \beta^{2 \alpha+1-2 s_{0}}+\kappa\|v\|_{L^{2}(\mathbb{R})} \beta^{-\frac{4 s-1}{2}} \\
& \quad+[v]_{K,\left(-\infty, x_{0}-\beta\right) \times\left(x_{0},+\infty\right)}^{2}+[v]_{K,\left(x_{0}-\beta, x_{0}\right) \times\left(x_{0}+\beta,+\infty\right)}^{2} .
\end{aligned}
$$

This and 7.7 imply that

$$
\begin{aligned}
& \quad\left|E_{\left(T_{1}, T_{2}\right)^{2}}(P)-E_{\left(T_{1}, x_{0}\right)^{2}}(P)-E_{\left(x_{0}, T_{2}\right)^{2}}(P)+2\left[Q_{\zeta_{1}, \zeta_{2}}^{\sharp}\right]_{K,\left(x_{0}-\beta, x_{0}\right) \times\left(x_{0}, x_{0}+\beta\right)}^{2}\right| \\
& \leqslant \kappa \delta^{2} \beta^{2 \alpha+1-2 s}+\kappa\|v\|_{L^{2}(\mathbb{R})} \beta^{-\frac{4 s-1}{2}} \\
& \quad+2[v]_{K,\left(-\infty, x_{0}-\beta\right) \times\left(x_{0},+\infty\right)}^{2}+2[v]_{K,\left(x_{0}-\beta, x_{0}\right) \times\left(x_{0}+\beta,+\infty\right)}^{2} .
\end{aligned}
$$

Now, thanks to 7.13 , one can consider a clean point $x_{0}$ (according to Definitions 7.1 and 7.2 and glue an optimal trajectory $Q_{\eta, \mu}$ to a linear interpolation with the equilibrium $\zeta$, close to $Q_{\eta, \mu}\left(x_{0}\right)$. Namely, one can consider

$$
P(x):=\left\{\begin{array}{cc}
Q_{\eta, \mu}(x) & \text { if } x \leqslant x_{0}, \\
R(x) & \text { if } x>x_{0}
\end{array}\right.
$$


where $R$ is such that $P-Q_{\zeta_{1}, \zeta_{2}}^{\sharp} \in H^{1}(\mathbb{R})$ and it is defined in $\left[x_{0}, x_{0}+\beta\right]$ as follows:

$$
R(x):=\left\{\begin{array}{cc}
Q_{\eta, \mu}\left(x_{0}\right)\left(x_{0}+1-x\right)+\zeta\left(x-x_{0}\right) & \text { if } x \in\left(x_{0}, x_{0}+1\right), \\
\zeta & \text { if } x \in\left[x_{0}+1, x_{0}+\beta\right) .
\end{array}\right.
$$

In this way, and taking $\rho>0$ suitably small, by Definitions 7.1 and 7.2 we know that $Q_{\eta, \mu}$ is $\rho$-close to an equilibrium in $\left[x_{0}-4 \beta, x_{0}+4 \beta\right]$, with

$$
\beta=\beta(\rho)=\frac{|\log \rho|}{8} .
$$

Moreover, by Lemma 7.2 we have that, for $\alpha \in(0,2 s)$,

$$
\left[Q_{\eta, \mu}\right]_{C^{0, \alpha}\left(x_{0}-\beta, x_{0}+\beta\right)} \leqslant C\left(\frac{\rho^{1-\frac{\alpha}{2 s}}}{|\log \rho|^{\alpha}}+\rho+\mu^{\frac{\alpha}{2 s}} \rho^{1-\frac{\alpha}{2 s}}\right),
$$

for some $C>0$. Also, we observe that

$$
[R]_{C^{0, \alpha}\left(x_{0}, x_{0}+\beta\right)} \leqslant \kappa \rho .
$$

As a consequence of this and (7.16), the function $P$ defined in 7.14 satisfies 7.6 with

$$
\delta:=C\left(\frac{\rho^{1-\frac{\alpha}{2 s}}}{|\log \rho|^{\alpha}}+\rho+\mu^{\frac{\alpha}{2 s}} \rho^{1-\frac{\alpha}{2 s}}\right)
$$

and $\alpha \in(0,2 s)$. Thus, choosing $\beta$ as in (7.15) and $\delta$ as in (7.17), and recalling (5.11) and (5.13), we infer from estimate 7.13 that

$$
\begin{aligned}
& \mid E_{\left(T_{1}, T_{2}\right)^{2}}(P)-E_{\left(T_{1}, x_{0}\right)^{2}}\left(Q_{\eta, \mu}\right) \\
& -E_{\left(x_{0}, T_{2}\right)^{2}}(R)+2\left[Q_{\zeta_{1}, \zeta_{2}}^{\sharp}\right]_{K,\left(x_{0}-\beta, x_{0}\right) \times\left(x_{0}, x_{0}+\beta\right)}^{2} \mid \leqslant \diamond,
\end{aligned}
$$

where we use the notation " $\diamond$ " to denote quantities that are as small as we wish when $\rho$ is sufficiently small. The smallness of $\rho$ depends on $\mu, Q_{\zeta_{1}, \zeta_{2}}^{\sharp}$ and the structural constants of the kernel and the potential, but it is independent on $\eta$.

We remark that, in virtue of (7.16), we also have that

$$
\begin{aligned}
& \mid E_{\left(T_{1}, T_{2}\right)^{2}}\left(Q_{\eta, \mu}\right)-E_{\left(T_{1}, x_{0}\right)^{2}}\left(Q_{\eta, \mu}\right) \\
& \quad-E_{\left(x_{0}, T_{2}\right)^{2}}\left(Q_{\eta, \mu}\right)+2\left[Q_{\zeta_{1}, \zeta_{2}}^{\sharp}\right]_{K,\left(x_{0}-\beta, x_{0}\right) \times\left(x_{0}, x_{0}+\beta\right)}^{2} \mid \leqslant \diamond .
\end{aligned}
$$

\section{Stickiness properties of energy minimizers}

In this section we show that the minimizers have the tendency to stick at the minima of $W$ once they arrive sufficiently close to them. For this, we recall that $r \in$ $\left(0, \min \left\{\delta_{0}, r_{0}\right\}\right]$ (with $r_{0}$ and $\delta_{0}$ as in (1.4) and (1.8), respectively) has been fixed at the beginning of Section 5 .

Proposition 8.1. Let $\rho \in(0,1)$. Let $Q_{\eta, \mu}$ be as in Lemma 5.1. Let $x_{1}, x_{2} \in \mathbb{R}$ be clean points for $\left(\rho, Q_{\eta, \mu}\right)$, according to Definition 7.2. with $x_{2} \geqslant x_{1}+4$, and

$$
\max _{i=1,2}\left|Q_{\eta, \mu}\left(x_{i}\right)-\zeta\right| \leqslant \rho,
$$


for some $\zeta \in \mathscr{Z}$. Then

$$
\begin{gathered}
\frac{\eta}{2} \int_{x_{1}}^{x_{2}}\left|\dot{Q}_{\eta, \mu}(x)\right|^{2} d x+\frac{\mu}{2} \int_{x_{1}}^{x_{2}}\left|Q_{\eta, \mu}(x)-Q_{\zeta_{1}, \zeta_{2}}^{\sharp}(x)\right|^{2} d x \\
+\frac{1}{4}\left[Q_{\eta, \mu}\right]_{K,\left(x_{1}, x_{2}\right)^{2}}^{2}+\int_{x_{1}}^{x_{2}} a(x) W\left(Q_{\eta, \mu}(x)\right) d x \leqslant \diamond,
\end{gathered}
$$

with $\diamond$ as small as we wish if $\rho$ is suitably small (the smallness of $\rho$ depends on $\mu$, $Q_{\zeta_{1}, \zeta_{2}}^{\sharp}$, and on structural constants, but it is independent on $\left.\eta\right)$.

Moreover,

$$
\left|Q_{\eta, \mu}(x)-\zeta\right| \leqslant \frac{r}{2} \text { for every } x \in\left[x_{1}, x_{2}\right] .
$$

Proof. First of all, we observe that, if $\rho$ is sufficiently small with respect to $\mu$, then

$$
\text { either } x_{2} \leqslant-2 \text { or } x_{1} \geqslant 2 \text {. }
$$

Indeed, suppose not, then $x_{2}>-2$ and $x_{1}<2$. Hence, if $x \in\left[x_{2}+3, x_{2}+\frac{|\log \rho|}{2}\right]$, we have that $x \geqslant 1$ and thus $Q_{\zeta_{1}, \zeta_{2}}^{\sharp}(x)=\zeta_{2}$. Consequently, recalling that $\mid Q_{\eta, \mu}(x)-$ $\zeta \mid \leqslant \rho$ for all $x \in\left[x_{2}+3, x_{2}+\frac{|\log \rho|}{2}\right]$, thanks to Definition 7.1 we find that

$$
\left|Q_{\eta, \mu}(x)-Q_{\zeta_{1}, \zeta_{2}}^{\sharp}(x)\right|=\left|Q_{\eta, \mu}(x)-\zeta_{2}\right| \geqslant\left|\zeta_{2}-\zeta\right|-\rho
$$

for all $x \in\left[x_{2}+3, x_{2}+\frac{|\log \rho|}{2}\right]$.

Similarly, we have that

$$
\left|Q_{\eta, \mu}(x)-Q_{\zeta_{1}, \zeta_{2}}^{\sharp}(x)\right| \geqslant\left|\zeta_{1}-\zeta\right|-\rho
$$

for all $x \in\left[x_{1}-\frac{|\log \rho|}{2}, x_{1}-3\right]$.

In particular, since either $\zeta \neq \zeta_{1}$ or $\zeta \neq \zeta_{2}$, we conclude that

$$
\left|Q_{\eta, \mu}(x)-Q_{\zeta_{1}, \zeta_{2}}^{\sharp}(x)\right| \geqslant c_{0},
$$

for some $c_{0}>0$, for all $x$ belonging to an interval of length $\frac{|\log \rho|}{2}-3 \geqslant \frac{|\log \rho|}{4}$.

For these reasons, we conclude that

$$
\left\|Q_{\eta, \mu}-Q_{\zeta_{1}, \zeta_{2}}^{\sharp}\right\|_{L^{2}(\mathbb{R})}^{2} \geqslant \frac{c_{0}^{2}|\log \rho|}{4} .
$$

This and $(5.13)$ yield that

$$
\frac{\kappa^{2}}{\mu^{2}} \geqslant\left\|Q_{\eta, \mu}-Q_{\zeta_{1}, \zeta_{2}}^{\sharp}\right\|_{L^{2}(\mathbb{R})}^{2} \geqslant \frac{c_{0}^{2}|\log \rho|}{4},
$$

which is a contradiction if $\rho$ is sufficiently small (possibly in dependence of $\mu$ ). This proves 8.4).

Hence, in the light of (8.4), without loss of generality we can now suppose that

$$
x_{1} \geqslant 2 \text {. }
$$


We claim that, in this case,

$$
\zeta=\zeta_{2}
$$

Indeed, suppose not. Then $\left|\zeta-\zeta_{2}\right| \geqslant \hat{c}$, for some $\hat{c}>0$. Also, if $x \geqslant x_{1}$ we have that $Q_{\zeta_{1}, \zeta_{2}}^{\sharp}(x)=\zeta_{2}$, and consequently, for all $x \in\left[x_{1}, x_{1}+\frac{|\log \rho|}{2}\right]$,

$$
\left|Q_{\eta, \mu}(x)-Q_{\zeta_{1}, \zeta_{2}}^{\sharp}(x)\right|=\left|Q_{\eta, \mu}(x)-\zeta_{2}\right| \geqslant\left|\zeta-\zeta_{2}\right|-\left|Q_{\eta, \mu}(x)-\zeta\right| \geqslant \hat{c}-\rho \geqslant \frac{\hat{c}}{2},
$$

if $\rho$ is small enough.

As a result,

$$
\left\|Q_{\eta, \mu}-Q_{\zeta_{1}, \zeta_{2}}^{\sharp}\right\|_{L^{2}(\mathbb{R})}^{2} \geqslant \frac{\hat{c}^{2}|\log \rho|}{8} .
$$

This and (5.13) yield that

$$
\frac{\kappa^{2}}{\mu^{2}} \geqslant \frac{\hat{c}^{2}|\log \rho|}{8}
$$

from which we obtain a contradiction if $\rho$ is small enough. The proof of 8.6 is thereby complete.

Now, in light of $(8.6)$, we define

$$
P(x):=\left\{\begin{array}{cc}
Q_{\eta, \mu}(x) & \text { if } x \in\left(-\infty, x_{1}\right), \\
Q_{\eta, \mu}\left(x_{1}\right)\left(x_{1}+1-x\right)+\zeta_{2}\left(x-x_{1}\right) & \text { if } x \in\left[x_{1}, x_{1}+1\right], \\
\zeta_{2} & \text { if } x \in\left(x_{1}+1, x_{2}-1\right), \\
Q_{\eta, \mu}\left(x_{2}\right)\left(x-x_{2}+1\right)+\zeta_{2}\left(x_{2}-x\right) & \text { if } x \in\left[x_{2}-1, x_{2}\right], \\
Q_{\eta, \mu}(x) & \text { if } x \in\left(x_{2},+\infty\right) .
\end{array}\right.
$$

In this way, we have that

$$
[P]_{C^{0,1}\left(x_{1}, x_{2}\right)} \leqslant \rho .
$$

Also, in view of 8.5,

$$
\begin{aligned}
\int_{x_{1}}^{x_{2}}\left|P(x)-Q_{\zeta_{1}, \zeta_{2}}^{\sharp}(x)\right|^{2} d x & =\int_{x_{1}}^{x_{2}}\left|P(x)-\zeta_{2}\right|^{2} d x \\
& =\int_{\left[x_{1}, x_{1}+1\right] \cup\left[x_{2}-1, x_{2}\right]}\left|P(x)-\zeta_{2}\right|^{2} d x .
\end{aligned}
$$

Moreover, we observe that, if $x \in\left(x_{1}, x_{2}\right)$, then

$$
\begin{aligned}
& \quad\left|P(x)-\zeta_{2}\right| \\
& \leqslant \sup _{y \in\left(x_{1}, x_{1}+1\right)}\left|Q_{\eta, \mu}\left(x_{1}\right)\left(x_{1}+1-y\right)+\zeta_{2}\left(y-x_{1}\right)-\zeta_{2}\right| \\
& \quad+\sup _{y \in\left(x_{2}-1, x_{2}\right)}\left|Q_{\eta, \mu}\left(x_{2}\right)\left(y-x_{2}-1\right)+\zeta_{2}\left(x_{2}-y\right)-\zeta_{2}\right| \\
& \quad \leqslant\left|Q_{\eta, \mu}\left(x_{1}\right)-\zeta_{2}\right|+\left|Q_{\eta, \mu}\left(x_{2}\right)-\zeta_{2}\right| \\
& \leqslant 2 \rho,
\end{aligned}
$$


thanks to 8.1 and (8.6).

Therefore, plugging this information into (8.8),

$$
\int_{x_{1}}^{x_{2}}\left|P(x)-Q_{\zeta_{1}, \zeta_{2}}^{\sharp}(x)\right|^{2} d x \leqslant 2 \rho^{2} .
$$

For that reason,

$$
\begin{aligned}
& \int_{\mathbb{R}}\left|P(x)-Q_{\zeta_{1}, \zeta_{2}}^{\sharp}(x)\right|^{2} d x-\int_{\mathbb{R}}\left|Q_{\eta, \mu}(x)-Q_{\zeta_{1}, \zeta_{2}}^{\sharp}(x)\right|^{2} d x \\
= & \int_{x_{1}}^{x_{2}}\left|P(x)-Q_{\zeta_{1}, \zeta_{2}}^{\sharp}(x)\right|^{2} d x-\int_{x_{1}}^{x_{2}}\left|Q_{\eta, \mu}(x)-Q_{\zeta_{1}, \zeta_{2}}^{\sharp}(x)\right|^{2} d x \\
\leqslant & 2 \rho^{2}-\int_{x_{1}}^{x_{2}}\left|Q_{\eta, \mu}(x)-Q_{\zeta_{1}, \zeta_{2}}^{\sharp}(x)\right|^{2} d x .
\end{aligned}
$$

Also,

$$
\text { if } x, y \in\left(x_{1}, x_{2}\right) \text {, then }|P(x)-P(y)| \leqslant 2 \rho \text {. }
$$

Now, let us estimate $[P]_{K,\left(x_{1}, x_{2}\right)^{2}}^{2}$. We have

$$
\begin{aligned}
{[P]_{K,\left(x_{1}, x_{2}\right)^{2}}^{2}=} & {[P]_{K,\left(x_{1}, x_{1}+1\right) \times\left(x_{1}, x_{2}\right)}^{2} } \\
& +[P]_{K,\left(x_{1}+1, x_{2}-1\right) \times\left(x_{1}, x_{2}\right)}^{2}+[P]_{K,\left(x_{2}-1, x_{2}\right) \times\left(x_{1}, x_{2}\right)}^{2} .
\end{aligned}
$$

Using (1.4), 8.7) and 8.11, we see that

$$
\begin{aligned}
& {[P]_{K,\left(x_{1}, x_{1}+1\right) \times\left(x_{1}, x_{2}\right)}^{2} } \\
= & \int_{x_{1}}^{x_{1}+1} \int_{x_{1}}^{x_{1}+2}|P(x)-P(y)|^{2} K(x-y) d x d y \\
& \quad+\int_{x_{1}}^{x_{1}+1} \int_{x_{1}+2}^{x_{2}}|P(x)-P(y)|^{2} K(x-y) d x d y \\
\leqslant & \Theta_{0} \rho^{2} \int_{x_{1}}^{x_{1}+1} \int_{x_{1}}^{x_{1}+2}|x-y|^{1-2 s} d x d y+4 \Theta_{0} \rho^{2} \int_{x_{1}}^{x_{1}+1} \int_{x_{1}+2}^{x_{2}}|x-y|^{-1-2 s} d x d y \\
\leqslant & \kappa \rho^{2} \\
= & \diamond .
\end{aligned}
$$

Similarly,

$$
[P]_{K,\left(x_{2}-1, x_{2}\right) \times\left(x_{1}, x_{2}\right)}^{2} \leqslant \diamond .
$$


Finally, making again use of (1.4), 8.7) and (8.11), we compute

$$
\begin{aligned}
& {[P]_{K\left(x_{1}+1, x_{2}-1\right) \times\left(x_{1}, x_{2}\right)}^{2} } \\
= & \int_{x_{1}+1}^{x_{2}-1} \int_{x_{1}}^{x_{1}+1}|P(x)-P(y)|^{2} K(x-y) d x d y \\
& \quad+\int_{x_{1}+1}^{x_{2}-1} \int_{x_{2}-1}^{x_{2}}|P(x)-P(y)|^{2} K(x-y) d x d y \\
= & \int_{x_{1}+1}^{x_{1}+2} \int_{x_{1}}^{x_{1}+1}|P(x)-P(y)|^{2} K(x-y) d x d y \\
& \quad+\int_{x_{1}+2}^{x_{2}-1} \int_{x_{1}}^{x_{1}+1}|P(x)-P(y)|^{2} K(x-y) d x d y \\
& \quad+\int_{x_{1}+1}^{x_{2}-2} \int_{x_{2}-1}^{x_{2}}|P(x)-P(y)|^{2} K(x-y) d x d y \\
& +\int_{x_{2}-2}^{x_{2}-1} \int_{x_{2}-1}^{x_{2}}|P(x)-P(y)|^{2} K_{m}(x-y) d x d y \\
\leqslant & \kappa \rho^{2}
\end{aligned}
$$

Therefore, collecting estimates 8.12, 8.13, 8.14 and 8.15, we get

$$
[P]_{K,\left(x_{1}, x_{2}\right)^{2}}^{2} \leqslant \diamond .
$$

Combining 7.18) (applied here twice, with $x_{0}:=x_{1}$ and $x_{0}:=x_{2}$ ) with 8.16 yields, for $\beta$ as in 7.15 ,

$$
\begin{aligned}
E_{\mathbb{R}^{2}}(P) \leqslant & E_{\left(-\infty, x_{1}\right)^{2}}\left(Q_{\eta, \mu}\right)+E_{\left(x_{1}, x_{2}\right)^{2}}(P)+E_{\left(x_{2},+\infty\right)^{2}}\left(Q_{\eta, \mu}\right)+\diamond \\
& \quad-2\left[Q_{\zeta_{1}, \zeta_{2}}^{\sharp}\right]_{K,\left(x_{1}-\beta, x_{1}\right) \times\left(x_{1}, x_{1}+\beta\right)}^{2}-2\left[Q_{\zeta_{1}, \zeta_{2}}^{\sharp}\right]_{K,\left(x_{2}-\beta, x_{2}\right) \times\left(x_{2}, x_{2}+\beta\right)}^{2} \\
= & E_{\left(-\infty, x_{1}\right)^{2}}\left(Q_{\eta, \mu}\right)+E_{\left(x_{2},+\infty\right)^{2}}\left(Q_{\eta, \mu}\right)+\diamond-\left[Q_{\zeta_{1}, \zeta_{2}}^{\sharp}\right]_{K,\left(x_{1}, x_{2}\right)^{2}}^{2} \\
& \quad-2\left[Q_{\zeta_{1}, \zeta_{2}}^{\sharp}\right]_{K,\left(x_{1}-\beta, x_{1}\right) \times\left(x_{1}, x_{1}+\beta\right)}^{2}-2\left[Q_{\zeta_{1}, \zeta_{2}}^{\sharp}\right]_{K,\left(x_{2}-\beta, x_{2}\right) \times\left(x_{2}, x_{2}+\beta\right)}^{2} .
\end{aligned}
$$

On the other hand, by 7.19 (again applied here twice, with $x_{0}:=x_{1}$ and $x_{0}:=x_{2}$ ), we have that

$$
\begin{aligned}
E_{\mathbb{R}^{2}}\left(Q_{\eta, \mu}\right) \geqslant & E_{\left(-\infty, x_{1}\right)^{2}}\left(Q_{\eta, \mu}\right)+E_{\left(x_{1}, x_{2}\right)^{2}}\left(Q_{\eta, \mu}\right)+E_{\left(x_{2},+\infty\right)^{2}}\left(Q_{\eta, \mu}\right)+\diamond \\
& -2\left[Q_{\zeta_{1}, \zeta_{2}}^{\sharp}\right]_{K,\left(x_{1}-\beta, x_{1}\right) \times\left(x_{1}, x_{1}+\beta\right)}^{2}-2\left[Q_{\zeta_{1}, \zeta_{2}}^{\sharp}\right]_{K,\left(x_{2}-\beta, x_{2}\right) \times\left(x_{2}, x_{2}+\beta\right)}^{2} .
\end{aligned}
$$


Subtracting (8.18) to 8.17), we get

$$
E_{\mathbb{R}^{2}}(P)-E_{\mathbb{R}^{2}}\left(Q_{\eta, \mu}\right) \leqslant-\left[Q_{\eta, \mu}\right]_{K\left(x_{1}, x_{2}\right)^{2}}^{2}+\diamond .
$$

In addition, by $(1.8)$ and $(8.9)$, we see that if $x \in\left(x_{1}, x_{2}\right)$ then $W(P(x)) \leqslant 4 C_{0} \rho^{2}$. Using this and the fact that $W(P(x))=W(\zeta)=0$ if $x \in\left(x_{1}+1, x_{2}-1\right)$, we conclude that

$$
\int_{x_{1}}^{x_{2}} W(P(x)) d x=\int_{x_{1}}^{x_{1}+1} W(P(x)) d x+\int_{x_{2}-1}^{x_{2}} W(P(x)) d x \leqslant 8 C_{0} \rho^{2} .
$$

Thus, by the minimality of $Q_{\eta, \mu}$ for $I_{\eta, \mu}$ (defined in 5.8 ), 8.10) and 8.19),

$$
\begin{aligned}
0 & \leqslant I_{\eta, \mu}(P)-I_{\eta, \mu}\left(Q_{\eta, \mu}\right) \\
\leqslant \eta \rho- & \frac{\eta}{2} \int_{x_{1}}^{x_{2}}\left|\dot{Q}_{\eta, \mu}(x)\right|^{2} d x+\mu \rho^{2}-\frac{\mu}{2} \int_{x_{1}}^{x_{2}}\left|Q_{\eta, \mu}(x)-Q_{\zeta_{1}, \zeta_{2}}^{\sharp}(x)\right|^{2} d x \\
& -\frac{1}{4}\left[Q_{\eta, \mu}\right]_{K,\left(x_{1}, x_{2}\right)^{2}}^{2}-\int_{x_{1}}^{x_{2}} a(x) W\left(Q_{\eta, \mu}(x)\right) d x+\diamond,
\end{aligned}
$$

which proves 8.2 .

Now we prove 8.3 . For this, we assume by contradiction that there exists $\tilde{x} \in$ $\left[x_{1}, x_{2}\right]$ such that $\left|Q_{\eta, \mu}(\tilde{x})-\zeta\right|>r / 2$.

By Corollary 6.1, we have that $Q_{\eta, \mu}$ is Hölder continuous (with uniform bound). Hence, since $\left|Q_{\eta, \mu}\left(x_{1}\right)-\zeta\right| \leqslant \rho<r / 2$, we obtain that there exists $\hat{x} \in\left[x_{1}, x_{2}\right]$ such that

$$
|Q(\hat{x})-\zeta|=\frac{r}{2} .
$$

In particular, there exists $\ell$ independent of $\eta$ and $\mu$ such that, for any $x \in[\hat{x}-\ell, \hat{x}+\ell]$ and $\alpha \in(0,2 s)$,

$$
\left|Q_{\eta, \mu}(x)-Q_{\eta, \mu}(\hat{x})\right| \leqslant \kappa|x-\hat{x}|^{\alpha} \leqslant \frac{r}{4} .
$$

This and 8.20 imply that, if $x \in[\hat{x}-\ell, \hat{x}+\ell]$,

$$
Q_{\eta, \mu}(x) \in \overline{B_{3 r / 4}(\zeta) \backslash B_{r / 4}(\zeta)}
$$

and thus

$$
\operatorname{dist}\left(Q_{\eta, \mu}(x), \mathscr{Z}\right) \geqslant \frac{r}{4},
$$

for all $x \in[\hat{x}-\ell, \hat{x}+\ell]$. This, $(1.8)$ and 1.10 give that

$$
\int_{\hat{x}-\ell}^{\hat{x}+\ell} a(x) W\left(Q_{\eta, \mu}(x)\right) d x \geqslant \underline{a} \int_{\hat{x}-\ell}^{\hat{x}+\ell} W\left(Q_{\eta, \mu}(x)\right) d x \geqslant 2 \ell \underline{a} \inf _{\operatorname{dist}(\tau, \mathscr{X}) \geqslant r / 4} W(\tau)=: c .
$$

Hence, noticing that $(\hat{x}-\ell, \hat{x}+\ell) \subseteq\left(x_{1}, x_{2}\right)$, we obtain that

$$
\int_{x_{1}}^{x_{2}} a(x) W\left(Q_{\eta, \mu}(x)\right) d x \geqslant c,
$$

and this is in contradiction with 8.2 for small $\rho$. Then, the proof of 8.3 is now complete. 


\section{Reduction to the case in which $\mathscr{Z}=\left\{\zeta_{1}, \zeta_{2}\right\}$}

Now we remark that it is enough to prove Theorem 1.1 under the additional assumption that

$$
\mathscr{Z}=\left\{\zeta_{1}, \zeta_{2}\right\} .
$$

Indeed, let $\zeta_{1} \neq \zeta_{2} \in \mathscr{Z}$ be nearest neighbors according to Definition 1.1, and suppose, without loss of generality, that $\zeta_{1}<\zeta_{2}$. Then, we can find $\delta>0$ sufficiently small such that $\mathscr{Z}^{\star}:=\left[\zeta_{1}-\delta, \zeta_{2}+\delta\right] \cap \mathscr{Z}=\left\{\zeta_{1}, \zeta_{2}\right\}$. We also consider a potential $W^{\star}$ which coincides with $W$ in $\left[\zeta_{1}-\delta, \zeta_{2}+\delta\right]$ and remains strictly positive outside $\left[\zeta_{1}-\right.$ $\left.\delta, \zeta_{2}+\delta\right]$.

In this way, the potential $W^{\star}$ satisfies the same structural assumptions of $W$ for a set of equilibria $\mathscr{Z}^{\star}$ as in (9.1). Then, if Theorem 1.1 holds true under the additional assumption (9.1), we obtain a heteroclinic orbit $Q^{\star}: \mathbb{R} \rightarrow\left[\zeta_{1}, \zeta_{2}\right]$ connecting $\zeta_{1}$ to $\zeta_{2}$, which is a solution of $\mathscr{L} Q^{\star}+a W^{\star \prime}\left(Q^{\star}\right)=0$. But then, since $W^{\star}=W$ in the range of $Q^{\star}$, we obtain that $Q^{\star}$ is also a solution of (1.13), thus giving Theorem 1.1 in its full generality.

For that reason, from now on, we assume without loss of generality that condition (9.1) is also satisfied.

\section{Unconstrained minimization for a perturbed problem}

Here, recalling the setting of Section 5, we show that if $b_{1}$ and $b_{2}$ are sufficiently separated, then the constrained minimizer, whose existence has been established in Lemma 5.1, is in fact an unconstrained minimizer. The idea for this is that the "excursion" of the minimizer will occur at the points "favored by the wells of $a$ " (recall the non-degeneracy condition in (1.12), which can be placed suitably far from the constraints.

Under the additional assumption in (9.1), we consider the minimizer $Q_{\eta, \mu}$ for $I_{\eta, \mu}$ as given in Lemma 5.1. In this setting, we have:

Proposition 10.1. There exists a structural constant $\mu_{0}>0$ such that if $\mu \in\left[0, \mu_{0}\right]$ the following statement holds true.

There exist $b_{1}, b_{2} \in \mathbb{R}$ and

$$
Q_{\eta, \mu}^{\star} \in \Gamma\left(b_{1}, b_{2}\right)
$$

such that

$$
Q_{\eta, \mu}^{\star}: \mathbb{R} \rightarrow\left[\min \left\{\zeta_{1}, \zeta_{2}\right\}, \max \left\{\zeta_{1}, \zeta_{2}\right\}\right]
$$

and

$$
I_{\eta, \mu}\left(Q_{\eta, \mu}^{\star}\right) \leqslant I_{\eta, \mu}(Q) \text { for all } Q \text { s.t. } Q-Q_{\zeta_{1}, \zeta_{2}}^{\sharp} \in H^{1}(\mathbb{R}) \text {. }
$$


Also, letting $v_{\eta, \mu}^{\star}:=Q_{\eta, \mu}^{\star}-Q_{\zeta_{1}, \zeta_{2}}^{\sharp}$, we see that

$$
\begin{aligned}
& {\left[v_{\eta, \mu}^{\star}\right]_{H^{1}(\mathbb{R})} \leqslant \frac{\kappa}{\sqrt{\eta \mu}}, } \\
& {\left[v_{\eta, \mu}^{\star}\right]_{K, \mathbb{R} \times \mathbb{R}} \leqslant \frac{\kappa}{\sqrt{\mu}}, } \\
& \left\|v_{\eta, \mu}^{\star}\right\|_{L^{\infty}(\mathbb{R})} \leqslant \kappa \\
& \left\|v_{\eta, \mu}^{\star}\right\|_{L^{2}(\mathbb{R})} \leqslant \frac{\kappa}{\mu} \\
\text { and } & \left\|v_{\eta, \mu}^{\star}\right\|_{C^{0, \alpha}(\mathbb{R})} \leqslant \kappa \text { for all } \alpha \in(0,2 s),
\end{aligned}
$$

for some $\kappa>0$, which possibly depends on $Q_{\zeta_{1}, \zeta_{2}}^{\sharp}$ and on structural constants.

Proof. We stress that the main difference between 5.9 and 10.3 is that the competitors in 10.3 do not need to be in $\Gamma\left(b_{1}, b_{2}\right)$ and so $Q_{\eta, \mu}^{\star}$ is a free minimizer. The proof of Proposition 10.1 is a slight modification of the proof of Theorem 9.4 in [21], and we refer to it for more details.

Let $Q_{\eta, \mu}^{\star}$ be as in Lemma 5.1 and let $v_{\eta, \mu}^{\star}:=Q_{\eta, \mu}^{\star}-Q_{\zeta_{1}, \zeta_{2}}^{\sharp}$. Then by Lemma 5.1 and Corollary 6.1 we have that $v_{\eta, \mu}^{\star}$ satisfies (10.4)-10.8).

To prove 10.3), we fix $\rho \in(0, r)$, to be taken sufficiently small, possibly in dependence of $\mu$, and we set

$$
b_{1}=m_{1} \quad \text { and } \quad b_{2}=m_{2},
$$

with $m_{1}, m_{2}$ given by 1.12 . To prove Proposition 10.1 we want to show that $Q_{\eta, \mu}^{\star}$ does not touch the constraints of $\Gamma\left(b_{1}, b_{2}\right)$. Assume by contradiction that

there exists $x_{1} \leqslant b_{1}=m_{1}$ such that either $Q_{\eta, \mu}^{\star}\left(x_{1}\right)=\Phi\left(x_{1}\right)$ or $Q_{\eta, \mu}^{\star}\left(x_{1}\right)=\Psi\left(x_{1}\right)$,

the other case being similar. In particular, by (5.5) and 5.6), we have that $\left|Q_{\eta, \mu}^{\star}\left(x_{1}\right)-\zeta_{1}\right| \geqslant \frac{3}{4} r$. Also, by 10.8 , we know that $\left[Q_{\eta, \mu}^{\star}\right]_{C^{0, \alpha}(\mathbb{R})} \leqslant \kappa$, for $\alpha \in(0,2 s)$. Thus, by Lemma 7.1 if

$$
\omega \geqslant \frac{\kappa_{1} \kappa^{\frac{1}{\alpha}}}{\mu^{2} \rho^{2+\frac{1}{\alpha}}}|\log \rho|+1
$$

we conclude that

there exist a clean point $x_{*} \in\left(m_{1}+1, m_{1}+\omega\right)$ and $\zeta \in \mathscr{Z}$ such that $Q_{\eta, \mu}^{\star}\left(x_{*}\right) \in \overline{B_{\rho}(\zeta)}$.

Furthermore, by (9.1), we have that $\zeta \in\left\{\zeta_{1}, \zeta_{2}\right\}$. Now, arguing as in the proof of Theorem 9.4 in [21] (see in particular the comments between (9.12) and (9.13) in $[21]$ ), and using $(10.9$, we see that we must actually have that

$$
\zeta=\zeta_{2}
$$


and that $Q_{\eta, \mu}^{\star}(x) \in \overline{B_{\frac{r}{2}}}\left(\zeta_{2}\right)$ for any $x \geqslant x_{*}$. In particular, since by 1.11, $x_{*} \leqslant$ $m_{1}+\omega \leqslant m_{2}-\theta$, we have that

$$
Q_{\eta, \mu}^{\star}(x) \in \overline{B_{\frac{r}{2}}}\left(\zeta_{2}\right) \text { for any } x \geqslant m_{2}-\theta .
$$

Now we define $P(x):=Q_{\eta, \mu}^{\star}(x-\theta)$. We remark that if $x \geqslant 1+\theta$ then $Q_{\zeta_{1}, \zeta_{2}}^{\sharp}(x)=$ $\zeta_{2}=Q_{\zeta_{1}, \zeta_{2}}^{\sharp}(x-\theta)$. Similarly, if $x \leqslant-1$, then $Q_{\zeta_{1}, \zeta_{2}}^{\sharp}(x)=\zeta_{1}=Q_{\zeta_{1}, \zeta_{2}}^{\sharp}(x-\theta)$. As a result, we see that the function $x \mapsto Q_{\zeta_{1}, \zeta_{2}}^{\sharp}(x-\theta)-Q_{\zeta_{1}, \zeta_{2}}^{\sharp}(x)$ vanishes outside $[-1,1+$ $\theta]$ and therefore

$$
\begin{aligned}
& \int_{\mathbb{R}}\left(\left|P(x)-Q_{\zeta_{1}, \zeta_{2}}^{\sharp}(x)\right|^{2}-\left|Q_{\eta, \mu}^{\star}(x)-Q_{\zeta_{1}, \zeta_{2}}^{\sharp}(x)\right|^{2}\right) d x \\
= & \int_{\mathbb{R}}\left(\left|Q_{\eta, \mu}^{\star}(x-\theta)-Q_{\zeta_{1}, \zeta_{2}}^{\sharp}(x)\right|^{2}-\left|Q_{\eta, \mu}^{\star}(x)-Q_{\zeta_{1}, \zeta_{2}}^{\sharp}(x)\right|^{2}\right) d x \\
= & \int_{\mathbb{R}}\left(\left|v_{\eta, \mu}^{\star}(x-\theta)+Q_{\zeta_{1}, \zeta_{2}}^{\sharp}(x-\theta)-Q_{\zeta_{1}, \zeta_{2}}^{\sharp}(x)\right|^{2}-\left|v_{\eta, \mu}^{\star}(x)\right|^{2}\right) d x \\
= & \int_{\mathbb{R}}\left(\left|v_{\eta, \mu}^{\star}(x-\theta)\right|^{2}-\left|v_{\eta, \mu}^{\star}(x)\right|^{2}+2 v_{\eta, \mu}^{\star}(x-\theta)\left(Q_{\zeta_{1}, \zeta_{2}}^{\sharp}(x-\theta)-Q_{\zeta_{1}, \zeta_{2}}^{\sharp}(x)\right)\right. \\
\qquad & \int_{\mathbb{R}}\left(2 v_{\eta, \mu}^{\star}(x-\theta)\left(Q_{\zeta_{1}, \zeta_{2}}^{\sharp}(x-\theta)-Q_{\zeta_{1}, \zeta_{2}}^{\sharp}(x-\theta)-\left.Q_{\zeta_{2}}^{\sharp}(x)\right|^{2}\right) d x\right. \\
\leqslant & \text { const } \left.\int_{\mathbb{R}}^{\sharp} \mid Q_{\zeta_{1}, \zeta_{2}}^{\sharp}(x)\right)+\left|Q_{\zeta_{1}, \zeta_{2}}^{\sharp}(x-\theta)-Q_{\zeta_{1}, \zeta_{2}}^{\sharp}(x)\right| d x \\
= & \text { const } \int_{-1}^{1+\theta}\left|Q_{\zeta_{1}, \zeta_{2}}^{\sharp}(x-\theta)-Q_{\zeta_{1}, \zeta_{2}}^{\sharp}(x)\right| d x \\
\leqslant & \text { const. }
\end{aligned}
$$

Also, due to 10.12, we have that $P \in \Gamma\left(b_{1}, b_{2}\right)$ and therefore, by 10.13 and the minimality of $Q_{\eta, \mu}^{\star}$,

$$
\begin{aligned}
0 \leqslant & I_{\eta, \mu}(P)-I_{\eta, \mu}\left(Q_{\eta, \mu}^{\star}\right) \\
= & \int_{\mathbb{R}} a(x) W(P(x)) d x-\int_{\mathbb{R}} a(x) W\left(Q_{\eta, \mu}^{\star}(x)\right) d x \\
& \quad+\frac{\mu}{2} \int_{\mathbb{R}}\left(\left|P(x)-Q_{\zeta_{1}, \zeta_{2}}^{\sharp}(x)\right|^{2}-\left|Q_{\eta, \mu}^{\star}(x)-Q_{\zeta_{1}, \zeta_{2}}^{\sharp}(x)\right|^{2}\right) d x \\
\leqslant & \int_{\mathbb{R}} a(x) W\left(Q_{\eta, \mu}^{\star}(x-\theta)\right) d x-\int_{\mathbb{R}} a(x) W\left(Q_{\eta, \mu}^{\star}(x)\right) d x+\operatorname{const} \mu \\
= & \int_{\mathbb{R}}[a(x+\theta)-a(x)] W\left(Q_{\eta, \mu}^{\star}(x)\right) d x+\operatorname{const} \mu .
\end{aligned}
$$

Now, we observe that $Q_{\eta, \mu}^{\star}\left(m_{1}\right) \in \overline{B_{\frac{5}{4} r}\left(\zeta_{1}\right)}$ and $Q_{\eta, \mu}^{\star}\left(x_{*}\right) \in \overline{B_{\rho}\left(\zeta_{2}\right)}$, due to 10.10 and 10.11. Therefore, since $Q_{\eta, \mu}^{*}$ is continuous, there exists $y_{*} \in\left(m_{1}, m_{1}+\omega\right)$ such that either $Q_{\eta, \mu}^{*}\left(y_{*}\right)=\zeta_{1}+\frac{1}{2}$ or $Q_{\eta, \mu}^{*}\left(y_{*}\right)=\zeta_{1}-\frac{1}{2}$. We assume without loss 
of generality that $Q_{\eta, \mu}^{*}\left(y_{*}\right)=\zeta_{1}+\frac{1}{2}$. Then by the Hölder continuity of $Q_{\eta, \mu}^{\star}$, there exists an interval $J_{*} \subset\left(m_{1}, m_{1}+\omega\right)$ of uniform length and centered at $y_{*}$ such that $Q_{\eta, \mu}^{\star}(x)$ stays at distance $1 / 4$ from $\mathscr{Z}$ for any $x \in J_{*}$. Therefore, using (1.12), we get

$$
\begin{gathered}
\int_{m_{1}-\omega}^{m_{1}+\omega}[a(x+\theta)-a(x)] W\left(Q_{\eta, \mu}^{\star}(x)\right) d x \leqslant \int_{J_{*}}[a(x+\theta)-a(x)] W\left(Q_{\eta, \mu}^{\star}(x)\right) d x \\
\leqslant-\gamma \int_{J_{*}} W\left(Q_{\eta, \mu}^{\star}(x)\right) d x \leqslant-\tilde{\gamma} \inf _{\operatorname{dist}(\tau, \mathscr{E}) \geqslant 1 / 4}=:-\hat{\gamma} .
\end{gathered}
$$

Then, by 10.7 and the continuity of $Q_{\eta, \mu}^{\star}$, we know that there exists a sequence of points $y_{k} \geqslant b_{2}=m_{2}$ with $y_{k} \rightarrow+\infty$ as $k \rightarrow+\infty$, such that $y_{k}$ is a clean point for $Q_{\eta, \mu}^{\star}$ and $Q_{\eta, \mu}^{\star}\left(y_{k}\right) \in \overline{B_{\rho}\left(\zeta_{2}\right)}$. Then, recalling 10.10 and 10.11, by 8.2 and $(1.10)$, we have that

$$
\int_{x_{*}}^{y_{k}}[a(x+\theta)-a(x)] W\left(Q_{\eta, \mu}^{\star}(x)\right) d x \leqslant \diamond .
$$

On that account, sending $k \rightarrow+\infty$, we obtain that

$$
\int_{m_{1}+\omega}^{+\infty}[a(x+\theta)-a(x)] W\left(Q_{\eta, \mu}^{\star}(x)\right) d x \leqslant \diamond .
$$

On the other hand, by arguing as in 21, we have that

$$
\int_{-\infty}^{m_{1}-\omega}[a(x+\theta)-a(x)] W\left(Q_{\eta, \mu}^{\star}(x)\right) d x \leqslant \diamond .
$$

By plugging 10.15, 10.16 and 10.17 into 10.14, we conclude that

$$
0 \leqslant-\hat{\gamma}+\diamond+\operatorname{const} \mu \text {. }
$$

Hence there exists a structural constant $\mu_{0}>0$ such that, if $\mu \in\left[0, \mu_{0}\right]$, then

$$
0 \leqslant-\frac{\hat{\gamma}}{2}+\diamond
$$

The latter inequality is negative for $\rho$ sufficiently small, and so we have obtained the desired contradiction. This proves 10.3.

\section{Vanishing viscosity method: sending $\eta \searrow 0$}

Now we consider the free minimizer constructed in Proposition 10.1 and we send $\eta \searrow$ 0 . The uniform estimates in (10.5), 10.6), 10.7) and (10.8) will allow us to pass to the limit and obtain a free minimizer, hence a solution, of a $\mu$-penalized nonlocal problem (then, in Section 13 , we will take the limit as $\mu \searrow 0$ of such a penalization).

This perturbative technique may be thought as a nonlocal counterpart of the socalled vanishing viscosity method for Hamilton-Jacobi equations, in which a small viscosity term is added as a perturbation to obtain solutions of the original equation. 
To this aim, we consider $I_{\mu}$ to be the energy functional corresponding to the choice $\eta:=0$ in (5.8), namely

$$
\begin{aligned}
I_{\mu}(Q):= & \frac{\mu}{2} \int_{\mathbb{R}}\left|Q(x)-Q_{\zeta_{1}, \zeta_{2}}^{\sharp}(x)\right|^{2} d x+\int_{\mathbb{R}} a(x) W(Q(x)) d x \\
& +\frac{1}{4} \iint_{\mathbb{R} \times \mathbb{R}}\left(|Q(x)-Q(y)|^{2}-\left|Q_{\zeta_{1}, \zeta_{2}}^{\sharp}(x)-Q_{\zeta_{1}, \zeta_{2}}^{\sharp}(y)\right|^{2}\right) K(x-y) d x d y .
\end{aligned}
$$

Then, for any $\eta>0$, we take $Q_{\eta, \mu}^{\star}$ to be the free minimizer given by Proposition 10.1 . We consider an infinitesimal sequence $\eta_{j} \searrow 0$ and let $Q_{j}^{\star}:=Q_{\eta_{j}, \mu}^{\star}$ and $v_{j}^{\star}:=$ $Q_{j}^{\star}-Q_{\zeta_{1}, \zeta_{2}}^{\sharp}$.

Since the estimates in 10.5, 10.6, 10.7) and 10.8) are uniform in $\eta_{j}$, up to a subsequence, for a fixed $\mu>0$ (suitably small to fulfill the assumption of Proposition 10.1), we can assume that $v_{j}^{\star}$ converges to some $v_{\mu}^{\star}$ locally uniformly in $\mathbb{R}$ and weakly in the Hilbert space induced by $[\cdot]_{K, \mathbb{R} \times \mathbb{R}}$. Then, we set $Q_{\mu}^{\star}:=v_{\mu}^{\star}+Q_{\zeta_{1}, \zeta_{2}}^{\sharp}$.

By taking limits in 10.3 , we obtain that

$$
I_{\mu}\left(Q_{\mu}^{\star}\right) \leqslant I_{\mu}(Q) \text { for all } Q \text { s.t. } Q-Q_{\zeta_{1}, \zeta_{2}}^{\sharp} \in H^{1}(\mathbb{R}) .
$$

As a consequence, differentiating the energy functional in 11.1) we find that

$$
\mu\left(Q-Q_{\zeta_{1}, \zeta_{2}}^{\sharp}\right)+\mathscr{L}(Q)(x)+a(x) W^{\prime}(Q(x))=0, \quad \text { for any } x \in \mathbb{R}
$$

in the distributional sense, and thus also in the viscosity sense, due to 40 .

Also, by 10.2 , we conclude that

$$
Q_{\mu}^{\star}: \mathbb{R} \rightarrow\left[\min \left\{\zeta_{1}, \zeta_{2}\right\}, \max \left\{\zeta_{1}, \zeta_{2}\right\}\right] .
$$

Moreover, in view of 10.1,

$$
Q_{\mu}^{\star} \in \Gamma\left(b_{1}, b_{2}\right)
$$

Finally, from 10.8 we obtain

$$
\left\|v_{\mu}^{\star}\right\|_{C^{0, \alpha}(\mathbb{R})} \leqslant \kappa \text { for all } \alpha \in(0,2 s) .
$$

\section{Asymptotics of solutions}

Here we discuss the spatial asymptotics of the solutions of 1.13 . In particular, we show that a solution which, at infinity, stays "close" to an equilibrium must converge to it.

Lemma 12.1. Let $\delta_{0}>0$ be as in (1.9). Let $b \in \mathbb{R}, \zeta \in \mathscr{Z}$ and $Q$ be a bounded solution of 1.13 in $\mathbb{R}$ such that

$$
\zeta-\delta_{0} \leqslant Q(x) \leqslant \zeta \quad \text { for all } x \geqslant b .
$$

Then

$$
\lim _{x \rightarrow+\infty} Q(x)=\zeta
$$


Proof. Let

$$
\lambda:=\liminf _{x \rightarrow+\infty} Q(x) .
$$

In view of 12.1], we have that $\lambda \in\left[\zeta-\delta_{0}, \zeta\right]$, and that the desired result is proven once we show that

$$
\lambda=\zeta .
$$

To prove this, suppose by contradiction that

$$
\lambda \in\left[\zeta-\delta_{0}, \zeta\right) .
$$

We take a diverging sequence $x_{k}$ such that

$$
\lim _{k \rightarrow+\infty} Q\left(x_{k}\right)=\lambda
$$

and we define $Q_{k}(x):=Q\left(x+x_{k}\right)$.

Since $\|Q\|_{C^{0, \alpha}(\mathbb{R})}<+\infty$ for some $\alpha \in(0,1)$, due to Theorem 12.1 in [10], we have that $Q_{k}$ is uniformly equicontinuous and therefore there exists a subsequence $Q_{k_{j}}$ that converges locally uniformly to some function $Q_{\infty}$. Then, by Corollary 4.7 in [10], we know that $Q_{\infty}$ is a viscosity solution of 1.13 in $\mathbb{R}$.

Notice also that, for all $x \in \mathbb{R}$,

$$
Q_{\infty}(x)=\lim _{j \rightarrow+\infty} Q_{k_{j}}(x)=\lim _{j \rightarrow+\infty} Q\left(x+x_{k_{j}}\right) \geqslant \liminf _{y \rightarrow+\infty} Q(y)=\lambda
$$

and

$$
Q_{\infty}(0)=\lim _{j \rightarrow+\infty} Q_{k_{j}}(0)=\lim _{j \rightarrow+\infty} Q\left(x_{k_{j}}\right)=\lambda,
$$

from which

$$
Q_{\infty}(x) \geqslant \lambda=Q_{\infty}(0) \quad \text { for all } x \in \mathbb{R} .
$$

Let

$$
w(x):= \begin{cases}\lambda & \text { in }(-1,1), \\ Q_{\infty}(x) & \text { in } \mathbb{R} \backslash(-1,1) .\end{cases}
$$

We stress that $w$ touches $Q_{\infty}$ from below at the origin, in view of (12.3), and consequently

$$
\mathscr{L} w(0)+a(0) W^{\prime}(\lambda) \geqslant 0 \text {. }
$$

On the other hand, by 1.3 and $(12.3)$,

$\mathscr{L} w(0)=$ P.V. $\int_{\mathbb{R}}(\lambda-w(y)) K(x-y) d y=\int_{\mathbb{R} \backslash(-1,1)}\left(\lambda-Q_{\infty}(y)\right) K(x-y) d y \leqslant 0$.

From this and (12.4), we conclude that $W^{\prime}(\lambda) \geqslant 0$. This is in contradiction with 1.9$)$, in light of 12.2 , and hence the proof is complete. 


\section{Sending $\mu \searrow 0$ and proof of Theorem 1.1}

We are now ready to complete the proof of Theorem 1.1. by taking the limit as $\mu \searrow 0$ of the penalized solution constructed in Section 11. To this end, we assume, for concreteness, that $\zeta_{2}>\zeta_{1}$. In light of 11.5 , up to a subsequence, we can suppose that $v_{\mu}^{\star}$ converges locally uniformly to a function $v^{\star}$. We set $Q^{\star}:=v^{\star}+Q_{\zeta_{1}, \zeta_{2}}^{\sharp}$. Notice that, by 11.3,

$$
Q^{\star}: \mathbb{R} \rightarrow\left[\zeta_{1}, \zeta_{2}\right]
$$

Furthermore, by Corollary 4.7 in 10 , we can also pass equation 11.2 to the limit, thus obtaining that $Q^{\star}$ is a solution of 1.13 .

We also remark that, by (11.4, we obtain that $Q^{\star} \in \Gamma\left(b_{1}, b_{2}\right)$. In particular, recalling (5.2), 5.6) and (5.7), we have that, for all $x \geqslant b_{2}+1$,

$$
Q^{\star}(x) \geqslant \Psi(x)=\psi(x)=\zeta_{2}-r .
$$

Thanks to this and (13.1), we are in the position of using Lemma 12.1 and thereby conclude that

$$
\lim _{x \rightarrow+\infty} Q^{\star}(x)=\zeta_{2}
$$

Similarly, one proves that

$$
\lim _{x \rightarrow-\infty} Q^{\star}(x)=\zeta_{1} .
$$

From this and 13.2, one obtains 1.14 , as desired. The proof of Theorem 1.1 is thus completed.

\section{Appendix A. Discontinuity and oscillatory behavior at infinity for functions in Sobolev spaces with low fractional exponents}

We recall here that functions belonging to the fractional Sobolev space $H^{s}(\mathbb{R})$ with $s \in\left(0, \frac{1}{2}\right)$ are not necessarily continuous, and they do not need to converge to zero at infinity.

To construct a simple example, let $\varphi \in C_{0}^{\infty}(\mathbb{R},[0,1])$ with $\varphi(0)=1$. Given a sequence $b_{k}$, let

$$
\varphi_{b_{k}}(x):=\varphi\left(e^{k}\left(x-b_{k}\right)\right) .
$$

Then

$$
\begin{aligned}
& \left\|\varphi_{b_{k}}\right\|_{L^{2}(\mathbb{R})}=\sqrt{\int_{\mathbb{R}}\left|\varphi\left(e^{k}\left(x-b_{k}\right)\right)\right|^{2} d x}=e^{-\frac{k}{2}} \sqrt{\int_{\mathbb{R}}|\varphi(X)|^{2} d X}=\text { const } e^{-\frac{k}{2}} \\
& \text { and }\left[\varphi_{b_{k}}\right]_{H^{s}(\mathbb{R})}=\sqrt{\iint_{\mathbb{R} \times \mathbb{R}} \frac{\left|\varphi\left(e^{k}\left(x-b_{k}\right)\right)-\varphi\left(e^{k}\left(y-b_{k}\right)\right)\right|^{2}}{|x-y|^{1+2 s}} d x d y} \\
& =e^{-\frac{(1-2 s) k}{2}} \sqrt{\iint_{\mathbb{R} \times \mathbb{R}} \frac{|\varphi(X)-\varphi(Y)|^{2}}{|X-Y|^{1+2 s}} d X d Y}=\operatorname{const} e^{-\frac{(1-2 s) k}{2}} .
\end{aligned}
$$


We now consider the superposition of the functions $\varphi_{b_{k}}$ with the choices $b_{k}:=k$ and $b_{k}:=1 / k$. Namely, if we set

$$
\Phi(x):=\sum_{k=1}^{+\infty} \varphi_{1 / k}(x)+\sum_{k=1}^{+\infty} \varphi_{k}(x),
$$

when $s \in\left(0, \frac{1}{2}\right)$ we have that

$$
\begin{aligned}
\|\Phi\|_{H^{s}(\mathbb{R})} & \leqslant \sum_{k=1}^{+\infty}\left\|\varphi_{1 / k}\right\|_{H^{s}(\mathbb{R})}+\sum_{k=1}^{+\infty}\left\|\varphi_{k}\right\|_{H^{s}(\mathbb{R})} \\
& \leqslant \text { const } \sum_{k=1}^{+\infty}\left(e^{-\frac{k}{2}}+e^{-\frac{(1-2 s) k}{2}}\right) \\
& \leqslant \text { const. }
\end{aligned}
$$

Nevertheless $\Phi$ is not continuous at the origin, and

$$
\limsup _{x \rightarrow+\infty} \Phi(x)>0=\liminf _{x \rightarrow+\infty} \Phi(x) .
$$

The case of $H^{1 / 2}(\mathbb{R})$ is slightly more delicate, since simple examples based on scaling, such as the one provided in (A.1), do not work in this case (and, in fact, functions in $H^{1 / 2}(\mathbb{R})$ have nicer properties in terms of topology than those in $H^{s}(\mathbb{R})$ with $s \in\left(0, \frac{1}{2}\right)$, see e.g. 5 ). Nevertheless, also functions in $H^{1 / 2}(\mathbb{R})$ are not necessarily continuous and they do not necessarily converge to zero at infinity. To construct an example of these behaviors, as depicted in Figure 1, we consider the function

$$
\mathbb{R}^{2} \ni X \mapsto \psi(X):=\left\{\begin{array}{cc}
\log (1-\log |X|) & \text { if } X \in B_{1} \backslash\{0\}, \\
0 & \text { otherwise. }
\end{array}\right.
$$

We claim that

$$
\psi \in H^{1}\left(\mathbb{R}^{2}\right)
$$

To check this, we notice that

$$
\psi \text { is supported in } B_{1},
$$

where we have that

$$
|\nabla \psi(X)|=\frac{1}{|X|(1-\log |X|)} .
$$

Therefore, using polar coordinates and the change of variable $t:=-\log \rho$, we find that

$$
\begin{gathered}
{[\psi]_{H^{1}(\mathbb{R})}^{2}=\int_{B_{1}} \frac{1}{|X|^{2}(1-\log |X|)^{2}} d X=2 \pi \int_{0}^{1} \frac{1}{\rho(1-\log \rho)^{2}} d \rho} \\
=2 \pi \int_{0}^{+\infty} \frac{1}{(1+t)^{2}} d t<+\infty
\end{gathered}
$$




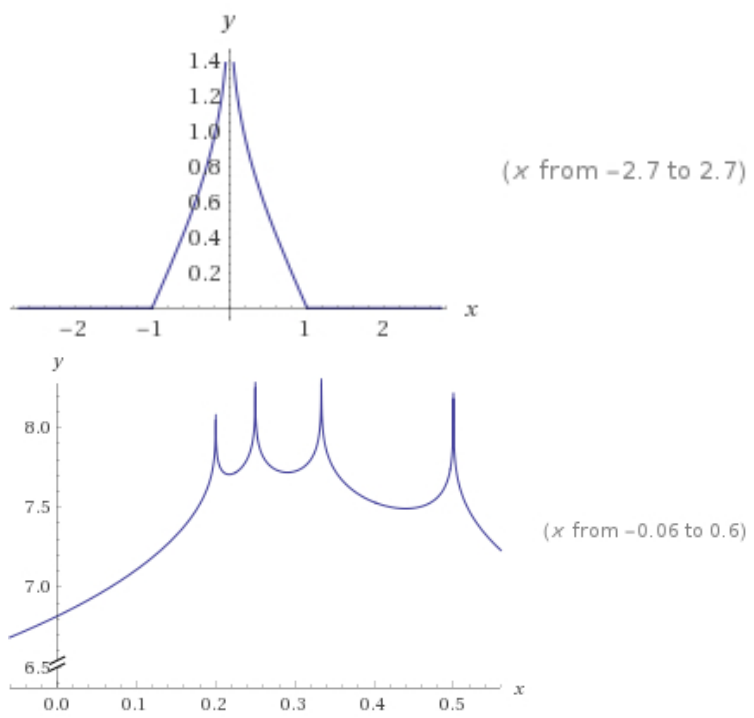

Figure 1. The function $\bar{\psi}$ and sketch of the construction of the function $\Psi$.

This, together with A.3 and the Poincaré inequality, proves A.2.

Then, from (A.2) and the Trace Theorem (see e.g. formula (3.19) in 19]), we obtain that

$$
\text { the function } \mathbb{R} \ni x \mapsto \bar{\psi}(x):=\psi(x, 0) \text { belongs to } H^{1 / 2}(\mathbb{R}) .
$$

Now we define the sequence of functions, for $k \in \mathbb{Z}$ and $X=(x, y) \in \mathbb{R} \times \mathbb{R}$,

$$
\psi_{k}(X)=\psi_{k}(x, y):=e^{-|k|} \bar{\psi}\left(e^{|k|}\left(x-e^{k}\right)\right) .
$$

Then, in view of A.4 we have that

$$
\begin{gathered}
\left\|\psi_{k}\right\|_{L^{2}(\mathbb{R})}=e^{-|k|} \sqrt{\int_{\mathbb{R}}\left|\bar{\psi}\left(e^{|k|}\left(x-e^{k}\right)\right)\right|^{2} d x}=e^{-\frac{3|k|}{2}} \sqrt{\int_{\mathbb{R}}|\bar{\psi}(\eta)|^{2} d \eta} \\
=e^{-\frac{3|k|}{2}}\|\bar{\psi}\|_{L^{2}(\mathbb{R})}=\mathrm{const} e^{-\frac{3|k|}{2}}
\end{gathered}
$$

and $\left[\psi_{k}\right]_{H^{1 / 2}(\mathbb{R})}=e^{-|k|} \sqrt{\iint_{\mathbb{R} \times \mathbb{R}} \frac{\left|\bar{\psi}\left(e^{|k|}\left(x-e^{k}\right)\right)-\bar{\psi}\left(e^{|k|}\left(y-e^{k}\right)\right)\right|^{2}}{|\bar{x}-\bar{y}|^{2}} d x d y}$

$$
=e^{-|k|} \sqrt{\iint_{\mathbb{R} \times \mathbb{R}} \frac{|\bar{\psi}(\eta)-\bar{\psi}(\xi)|^{2}}{|\eta-\xi|^{2}} d \eta d \xi}=e^{-|k|}[\bar{\psi}]_{H^{1 / 2}(\mathbb{R})}=\text { const } e^{-|k|} .
$$

Consequently, if we set

$$
\mathbb{R} \mapsto \Psi(x):=\sum_{k \in \mathbb{Z}} \psi_{k}(x)
$$


it follows that $\Psi$ is not continuous (and not even locally bounded) and it does not go to zero at infinity, but it belongs to $H^{1 / 2}(\mathbb{R})$ since

$$
\|\Psi\|_{H^{1 / 2}(\mathbb{R})} \leqslant \sum_{k \in \mathbb{Z}}\left\|\psi_{k}\right\|_{H^{1 / 2}(\mathbb{R})} \leqslant \text { const } \sum_{k \in \mathbb{Z}}\left(\text { const } e^{-\frac{3|k|}{2}}+\text { const } e^{-|k|}\right) \leqslant \text { const. }
$$

\section{Acknowledgments}

This work has been carried out during a very pleasant visit of the first and third authors to the University of Texas. Supported by the Australian Research Council Discovery Project grant "N.E.W. Nonlocal Equations at Work" and by the DECRA Project grant "Partial differential equations, free boundaries and applications". The authors are members of G.N.A.M.P.A.-I.N.d.A.M. We thank the anonymous Referees for their very useful remarks.

\section{References}

[1] Roberto Alicandro, Andrea Braides, and Marco Cicalese, Phase and anti-phase boundaries in binary discrete systems: a variational viewpoint, Netw. Heterog. Media 1 (2006), no. 1, 85-107, DOI 10.3934/nhm.2006.1.85. MR2219278

[2] Sayah Awatif, Équations d'Hamilton-Jacobi du premier ordre avec termes intégrodifférentiels. II. Existence de solutions de viscosité, Comm. Partial Differential Equations 16 (1991), no. 6-7, 1075-1093, DOI 10.1080/03605309108820790 (French). MR1116854

[3] Guy Barles, Emmanuel Chasseigne, and Cyril Imbert, Hölder continuity of solutions of second-order non-linear elliptic integro-differential equations, J. Eur. Math. Soc. (JEMS) 13 (2011), no. 1, 1-26, DOI 10.4171/JEMS/242. MR2735074

[4] Richard F. Bass and Moritz Kassmann, Hölder continuity of harmonic functions with respect to operators of variable order, Comm. Partial Differential Equations 30 (2005), no. 7-9, 12491259, DOI 10.1080/03605300500257677. MR2180302

[5] H. Brezis and L. Nirenberg, Degree theory and BMO. I. Compact manifolds without boundaries, Selecta Math. (N.S.) 1 (1995), no. 2, 197-263, DOI 10.1007/BF01671566. MR1354598

[6] Claudia Bucur and Enrico Valdinoci, Nonlocal diffusion and applications, Lecture Notes of the Unione Matematica Italiana, vol. 20, Springer, [Cham]; Unione Matematica Italiana, Bologna, 2016. MR3469920

[7] Xavier Cabré and Yannick Sire, Nonlinear equations for fractional Laplacians II: Existence, uniqueness, and qualitative properties of solutions, Trans. Amer. Math. Soc. 367 (2015), no. 2, 911-941, DOI 10.1090/S0002-9947-2014-05906-0. MR3280032

[8] Xavier Cabré and Joan Solà-Morales, Layer solutions in a half-space for boundary reactions, Comm. Pure Appl. Math. 58 (2005), no. 12, 1678-1732, DOI 10.1002/cpa.20093. MR2177165

[9] Luis Caffarelli, Serena Dipierro, and Enrico Valdinoci, A logistic equation with nonlocal interactions, Kinet. Relat. Models 10 (2017), no. 1, 141-170, DOI 10.3934/krm.2017006. MR3579567

[10] Luis Caffarelli and Luis Silvestre, Regularity theory for fully nonlinear integro-differential equations, Comm. Pure Appl. Math. 62 (2009), no. 5, 597-638, DOI 10.1002/cpa.20274. MR2494809

[11] Luis A. Caffarelli and Panagiotis E. Souganidis, Convergence of nonlocal threshold dynamics approximations to front propagation, Arch. Ration. Mech. Anal. 195 (2010), no. 1, 1-23, DOI 10.1007/s00205-008-0181-x. MR2564467 
[12] Robert Stephen Cantrell, Chris Cosner, Yuan Lou, and Daniel Ryan, Evolutionary stability of ideal free dispersal strategies: a nonlocal dispersal model, Can. Appl. Math. Q. 20 (2012), no. 1, 15-38. MR3026598

[13] K.-S. Chen, C. B. Muratov, and X. Yan, Layer solutions for a one-dimensional nonlocal model of Ginzburg-Landau type, Math. Model. Nat. Phenom. 12 (2017), no. 6, 68-90, DOI 10.1051/mmnp/2017068. MR3746096

[14] Chris Cosner, Juan Dávila, and Salome Martínez, Evolutionary stability of ideal free nonlocal dispersal, J. Biol. Dyn. 6 (2012), no. 2, 395-405, DOI 10.1080/17513758.2011.588341. MR2897881

[15] Matteo Cozzi, Juan Dávila, and Manuel del Pino, Long-time asymptotics for evolutionary crystal dislocation models, arXiv e-prints (2019), available at 1907.01491

[16] Matteo Cozzi, Serena Dipierro, and Enrico Valdinoci, Nonlocal phase transitions in homogeneous and periodic media, J. Fixed Point Theory Appl. 19 (2017), no. 1, 387-405, DOI 10.1007/s11784-016-0359-z. MR3625078

[17] Matteo Cozzi and Tommaso Passalacqua, One-dimensional solutions of non-local AllenCahn-type equations with rough kernels, J. Differential Equations 260 (2016), no. 8, 66386696, DOI 10.1016/j.jde.2016.01.006. MR3460227

[18] Michael G. Crandall, Hitoshi Ishii, and Pierre-Louis Lions, User's guide to viscosity solutions of second order partial differential equations, Bull. Amer. Math. Soc. (N.S.) 27 (1992), no. 1, 1-67, DOI 10.1090/S0273-0979-1992-00266-5. MR1118699

[19] Eleonora Di Nezza, Giampiero Palatucci, and Enrico Valdinoci, Hitchhiker's guide to the fractional Sobolev spaces, Bull. Sci. Math. 136 (2012), no. 5, 521-573, DOI 10.1016/j.bulsci.2011.12.004. MR2944369

[20] Serena Dipierro, Giampiero Palatucci, and Enrico Valdinoci, Dislocation dynamics in crystals: a macroscopic theory in a fractional Laplace setting, Comm. Math. Phys. 333 (2015), no. 2, 1061-1105, DOI 10.1007/s00220-014-2118-6. MR3296170

[21] Serena Dipierro, Stefania Patrizi, and Enrico Valdinoci, Chaotic orbits for systems of nonlocal equations, Comm. Math. Phys. 349 (2017), no. 2, 583-626, DOI 10.1007/s00220-016-2713-9. MR3594365

[22] Jack Dockery, Vivian Hutson, Konstantin Mischaikow, and Mark Pernarowski, The evolution of slow dispersal rates: a reaction diffusion model, J. Math. Biol. 37 (1998), no. 1, 61-83, DOI 10.1007/s002850050120. MR1636644

[23] A. Z. Fino, H. Ibrahim, and R. Monneau, The Peierls-Nabarro model as a limit of a Frenkel-Kontorova model, J. Differential Equations 252 (2012), no. 1, 258-293, DOI 10.1016/j.jde.2011.08.007. MR2852206

[24] N.E. Humphries, N. Queiroz, J. R. M. Dyer, N. G. Pade, M. K. Musyl, K. M. Schaefer, D. W. Fuller, J. M. Brunnschweiler, T. K. Doyle, J. D. R. Houghton, G. C. Hays, C. S. Jones, L. R. Noble, V. J. Wearmouth, E. J. Southall, and D. W. Sims, Environmental context explains Lévy and Brownian movement patterns of marine predators, Nature 465 (2010), 1066-1069.

[25] H. Ishii and P.-L. Lions, Viscosity solutions of fully nonlinear second-order elliptic partial differential equations, J. Differential Equations 83 (1990), no. 1, 26-78, DOI 10.1016/00220396(90)90068-Z. MR1031377

[26] Chiu-Yen Kao, Yuan Lou, and Wenxian Shen, Random dispersal vs. non-local dispersal, Discrete Contin. Dyn. Syst. 26 (2010), no. 2, 551-596, DOI 10.3934/dcds.2010.26.551. MR2556498

[27] Hans Lewy and Guido Stampacchia, On the smoothness of superharmonics which solve a minimum problem, J. Analyse Math. 23 (1970), 227-236. MR0271383

[28] Annalisa Massaccesi and Enrico Valdinoci, Is a nonlocal diffusion strategy convenient for biological populations in competition?, J. Math. Biol. 74 (2017), no. 1-2, 113-147, DOI 10.1007/s00285-016-1019-z. MR3590678 
[29] Régis Monneau and Stefania Patrizi, Homogenization of the Peierls-Nabarro model for dislocation dynamics, J. Differential Equations 253 (2012), no. 7, 2064-2105, DOI 10.1016/j.jde.2012.06.019. MR2946964

[30] F. R. N. Nabarro, Dislocations in Solids. The Elastic Theory, Vol. 1, North-Holland Publishing Company, Oxford, 1979.

[31] Giampiero Palatucci, Ovidiu Savin, and Enrico Valdinoci, Local and global minimizers for a variational energy involving a fractional norm, Ann. Mat. Pura Appl. (4) 192 (2013), no. 4, 673-718, DOI 10.1007/s10231-011-0243-9. MR3081641

[32] Paul H. Rabinowitz, Periodic and heteroclinic orbits for a periodic Hamiltonian system, Ann. Inst. H. Poincaré Anal. Non Linéaire 6 (1989), no. 5, 331-346 (English, with French summary). MR1030854

[33] _ Heteroclinics for a reversible Hamiltonian system, Ergodic Theory Dynam. Systems 14 (1994), no. 4, 817-829, DOI 10.1017/S0143385700008178. MR1304144

[34] Paul H. Rabinowitz and Vittorio Coti Zelati, Multichain-type solutions for Hamiltonian systems, Proceedings of the Conference on Nonlinear Differential Equations (Coral Gables, FL, 1999), Electron. J. Differ. Equ. Conf., vol. 5, Southwest Texas State Univ., San Marcos, TX, 2000, pp. 223-235. MR1799055

[35] Paul H. Rabinowitz, Connecting orbits for a reversible Hamiltonian system, Ergodic Theory Dynam. Systems 20 (2000), no. 6, 1767-1784, DOI 10.1017/S0143385700000985. MR1804957

[36] A. M. Reynolds, Lévy flight movement patterns in marine predators may derive from turbulence cues, Proc. R. Soc. Lond. Ser. A Math. Phys. Eng. Sci. 470 (2014), no. 2171, 20140408, 8, DOI 10.1098/rspa.2014.0408. MR3272943

[37] Xavier Ros-Oton and Joaquim Serra, The Dirichlet problem for the fractional Laplacian: regularity up to the boundary, J. Math. Pures Appl. (9) 101 (2014), no. 3, 275-302, DOI 10.1016/j.matpur.2013.06.003 (English, with English and French summaries). MR3168912

[38] Ovidiu Savin and Enrico Valdinoci, $\Gamma$-convergence for nonlocal phase transitions, Ann. Inst. H. Poincaré Anal. Non Linéaire 29 (2012), no. 4, 479-500, DOI 10.1016/j.anihpc.2012.01.006. MR2948285

[39] Raffaella Servadei and Enrico Valdinoci, Lewy-Stampacchia type estimates for variational inequalities driven by (non)local operators, Rev. Mat. Iberoam. 29 (2013), no. 3, 1091-1126, DOI 10.4171/RMI/750. MR3090147

[40] _ Weak and viscosity solutions of the fractional Laplace equation, Publ. Mat. 58 (2014), no. 1, 133-154. MR3161511

[41] Luis Enrique Silvestre, Regularity of the obstacle problem for a fractional power of the Laplace operator, ProQuest LLC, Ann Arbor, MI, 2005. Thesis (Ph.D.)-The University of Texas at Austin. MR2707618

[42] Jürgen Sprekels and Enrico Valdinoci, A new type of identification problems: optimizing the fractional order in a nonlocal evolution equation, SIAM J. Control Optim. 55 (2017), no. 1, 70-93, DOI 10.1137/16M105575X. MR3590646

[43] Elias M. Stein, Singular integrals and differentiability properties of functions, Princeton Mathematical Series, No. 30, Princeton University Press, Princeton, N.J., 1970. MR0290095

[44] J. F. Toland, The Peierls-Nabarro and Benjamin-Ono equations, J. Funct. Anal. 145 (1997), no. 1, 136-150, DOI 10.1006/jfan.1996.3016. MR1442163

[45] G. M. Viswanathan, V. Afanasyev, S. V. Buldyrev, E. J. Murphy, P. A. Prince, and H. E. Stanley, Lévy flight search patterns of wandering albatrosses, Nature 381 (1996), no. 1, $413-415$. 